\title{
Design, synthesis, and cytostatic activity of novel pyrazine sorafenib analogs
}

\author{
Zrinka Rajić Džolić $^{1}$ - Ivana Perković ${ }^{1}$ Sandra Kraljević Pavelić ${ }^{2} \cdot$ Mirela Sedić $^{2}$ • \\ Nataša Ilić ${ }^{2} \cdot$ Dominique Schols ${ }^{3} \cdot$ Branka Zorc $^{1}$
}

Received: 24 September 2015 / Accepted: 4 July 2016 / Published online: 17 August 2016

(C) The Author(s) 2016; This article is published with open access at Springerlink.com

\begin{abstract}
The current study is focused on a series of sorafenib analogs as potential antitumor agents. We have designed and synthesized nine novel pyrazine analogs $6 \mathbf{6}-\mathbf{i}$ differing in amide and/or urea regions. Two alternative strategies for the preparation of title compounds were applied. The first strategy involved ether formation between 4-hydroxyphenyl urea $\mathbf{3}$ and 5-chloro-pyrazine-2-carboxamides 4 . In the second strategy, ether functionality was introduced in the molecule before urea moiety and included preparation of 5-(4-aminophenoxy)- $N$-alkylpyrazine-2-carboxamides 5 and their reaction with 4-chloro-3-(fluoromethyl)phenyl isocyanate. Cytostatic activity of the title compounds was evaluated in vitro against a panel of cancer cell lines. Most of the tested compounds showed strong antiproliferative activity in the low micromolar range. 5-/4-[3-(4-chloro-3-trifluoromethyl-phenyl)-ureido]-phenoxy/pyrazine-2-carboxylic acid (4-chloro-3-trifluoromethylphenyl)amide $(\mathbf{6 g})$ was the most active compound $\left(\mathrm{IC}_{50} 0.9-7.5 \mu \mathrm{M}\right)$ and showed comparable or stronger activity than sorafenib, but also similar cytotoxicity to normal human fibroblast cells. Two compounds, namely, 5-/4-[3-(4-bromophenyl)-
\end{abstract}

Electronic supplementary material The online version of this article (doi:10.1007/s00044-016-1667-y) contains supplementary material, which is available to authorized users.

Branka Zorc

bzorc@pharma.hr

1 Faculty of Pharmacy and Biochemistry, University of Zagreb, A. Kovačića 1, HR-10 000 Zagreb, Croatia

2 Department of Biotechnology, University of Rijeka, Radmile Matejčić 2, HR-51 000 Rijeka, Croatia

3 Rega Institute for Medical Research, Katholieke Universiteit Leuven, Minderbroedersstraat 10, B-3000 Leuven, Belgium ureido]-phenyloxy/-pyrazine-2-carboxylic acid cyclopentylamide (6c) and 5-/4-[3-(4-chloro-3-trifluoromethyl-phenyl)ureido]-phenoxy/-pyrazine-2-carboxylic acid cyclopentylamide (6h), exerted cytostatic activities that surpassed the effects observed with sorafenib in three cancer cell lines (HepG2, HeLa, A549, IC $_{50} 0.6-0.9 \mu \mathrm{M}$ ). Similar to sorafenib, compound $\mathbf{6 h}$ proved to be cytotoxic to normal human fibroblast cells, whereas compound $\mathbf{6 c}$ did not diminish proliferative capacity of these cells and could be regarded as the most promising derivative. Additional biological studies on the c-Raf activity using Western blot method revealed that antiproliferative activity of $\mathbf{6 h}$ could be at least partially attributed to its inhibitory effect on c-Raf activation similar to sorafenib. In contrast, $\mathbf{6 c}$ did not inhibit the activity of c-Raf, which implies that other cell signaling pathways govern its antiproliferative effects. Taking into account structural differences between compounds $\mathbf{6 c}$ and $\mathbf{6 h}$, it is plausible to believe that the substituent in urea part of the molecule is essential for the interaction with c-Raf.

Keywords Sorafenib $\cdot$ Pyrazine $\cdot$ Synthesis $\cdot$ Cytostatic activity $\cdot \mathrm{c}$-Raf $\cdot$ Western blotting

$\begin{array}{ll}\text { Abbreviations } \\ \text { A549 } & \text { lung adenocarcinoma } \\ \text { BSA } & \text { bovine serum albumin } \\ \text { BtcCl } & \text { 1-benzotriazole carboxylic acid chloride } \\ \text { BtH } & \text { 1H-benzo[d][1,2,3]triazole } \\ \text { CEM } & \text { acute lymphoblastic leukemia } \\ \text { DMEM } & \text { Dulbecco's modified Eagle's medium } \\ \text { EDTA } & \text { ethylenediamine tetraacetic acid } \\ \text { EGTA } & \text { ethyleneglycol tetraacetic acid } \\ \text { FBS } & \text { foetal bovine serum } \\ \text { HeLa } & \text { cervical carcinoma }\end{array}$




\begin{tabular}{|c|c|}
\hline HepG2 & hepatocellular carcinoma \\
\hline $\mathrm{IC}_{50}$ & $\begin{array}{l}\text { the concentration that causes } 50 \% \text { growth } \\
\text { inhibition }\end{array}$ \\
\hline $\mathrm{KO}_{t} \mathrm{Bu}$ & potassium $t$-butoxide \\
\hline L1210 & murine lymphocytic leukemia \\
\hline MCF-7 & breast carcinoma \\
\hline Molt4/C8 & acute lymphoblastic leukemia \\
\hline MR & molecular refractivity \\
\hline MTT & $\begin{array}{l}\text { 3-(4,5-dimethylthiazol-2-yl)-2,5-diphenyltetra- } \\
\text { zolium bromide }\end{array}$ \\
\hline PG & percentage growth \\
\hline PSA & polar surface area \\
\hline PVDF & polyvinylidene fluoride \\
\hline c-Raf & $\begin{array}{l}\text { RAF proto-oncogene serine/threonine-protein } \\
\text { kinase }\end{array}$ \\
\hline RIPA & radioimmunoprecipitation assay buffer \\
\hline SW620 & metastatic colorectal adenocarcinoma \\
\hline TBST & Tris-buffered saline with Tween 20 \\
\hline TEA & triethylamine \\
\hline WI-38 & normal human diploid fibroblasts \\
\hline
\end{tabular}

\section{Introduction}

Cancer is one of the major health concerns in the world that represents the leading cause of death worldwide as evidenced by 8.2 million cancer-related deaths in 2012 (WHO, 2015). Because of high cancer morbidity and mortality, new approaches in cancer therapy are needed that require development of novel, more effective antitumor agents (Chong and Jänne, 2013; Holohan et al., 2013). Modification of known, clinically approved drugs, is one of the possible strategies in drug discovery. In the present study, we have directed our attention to sorafenib, the first multikinase inhibitor approved in 2005 for the therapy of advanced renal cell carcinoma and hepatocellular carcinoma. Sorafenib supresses tumor growth through the inhibition of C-RAF and B-RAF serine/threonine kinase activities and tumor angiogenesis by abrogating vascular endothelial growth factor receptor (VEGFR) and plateletderived growth factor receptors signaling (Wilhelm et al., 2006, 2008). Because of its multi-antitumoral mechanisms, a broad spectrum of anticancer activity, good results in combination trials, poor physicochemical properties, inactivity against mutated BRAF, toxicity and drug resistance (Schutz et al., 2011; Villaneuva and Llovet, 2012), many research efforts have been focused on the optimization of sorafenib molecule (Sun et al., 2010; Yao et al., 2012; Babić et al., 2012; Zhan et al., 2012; Lu et al., 2014; Qin et al., 2014; Jiao et al., 2015). As a result, a closely related analog of sorafenib, regorafenib, was registered in 2012, and is currently approved for the treatment of metastatic colorectal cancer and advanced gastrointestinal stromal tumors (Crona et al., 2013).

The widespread use of pyrazine heterocycle as an important pharmacophore in medicinal chemistry establishes this moiety as a member of a privileged structures class. As a part of ongoing studies focused on the development of new heterocycle anticancer agents, novel sorafenib analogs bearing pyrazine scaffold were prepared. Herein, we report their synthesis, chemical characterization, in vitro screening, and the effects on the activity of known sorafenib target, namely, c-Raf kinase.

\section{Results and discussion}

\section{Chemistry}

Novel compounds 6a-i described in this paper are derived from the cytostatic drug sorafenib. Crucial parts of the structure (diarylurea, ether, heterocycle, amide) are preserved, but a different heterocycle (pyrazine instead of pyridine) and various substituents in urea and/or amide regions were introduced. Comparison of the title sorafenib analogs and the parent molecule is given in Fig. 1.

A pyridine heterocycle was replaced by its isoster pyrazine in the hope that the additional nitrogen atom would increase the binding interactions between the drug and c-Raf kinase. The central bis-aryl urea and the amide functionalities were left intact, since it was shown that they played key roles in the interaction with Raf (Wan et al., 2004), but various substituents in these two regions were introduced.

Majority of the pyrazine sorafenib analogs are fully in agreement with the Lipinski's and Gelovani's rules for prospective small molecular drugs ( $\mathrm{MW} \leq 500, \log P \leq 5$, number of H-bond donors $\leq 5$, number of $\mathrm{H}$-bond acceptors $\leq 10$, molecular polar surface area $<140 \AA^{2}$, molar refractivity within the range of 40 and $130 \mathrm{~cm}^{3} / \mathrm{mol}$, the number of atoms 20-70) and some analogs showed minimal aberrations of the rules (Luzina and Popov, 2012). The parameters are calculated with Chemicalize.org program and presented in Table 1.

Two alternative strategies for the preparation of the title compounds 6a-i were applied (Scheme 1). The first strategy involved ether formation between 4-hydroxyphenyl urea 3a-c and 5-chloro-pyrazine-2-carboxamides 4a-f. The reactions were performed in dimethylformamide (DMF) in the presence of $\mathrm{K}_{2} \mathrm{CO}_{3}$. Ureas 3 were prepared from $\mathrm{N}$-(4hydroxyphenyl)- $1 H$-benzo[ $d][1,2,3]$ triazole-1-carboxamide (2) and the corresponding amine [cyclopentylamine, 4-bromoaniline or 4-chloro-3-(trifluoromethyl)aniline], while compound $\mathbf{2}$ was synthesized from $\mathrm{BtcCl}$ (1) and 
Fig. 1 Comparison of sorafenib analogs $\mathbf{6 a}-\mathbf{i}$ and the parent molecule. The regions exposed to changes are cycled

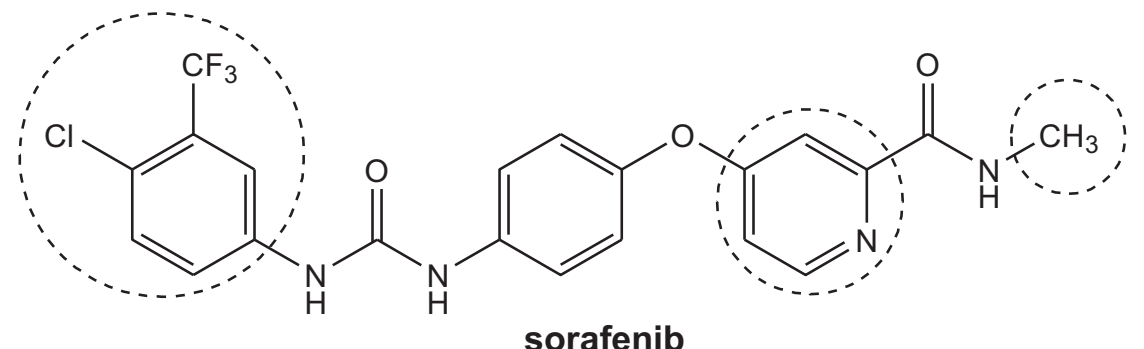

sorafenib

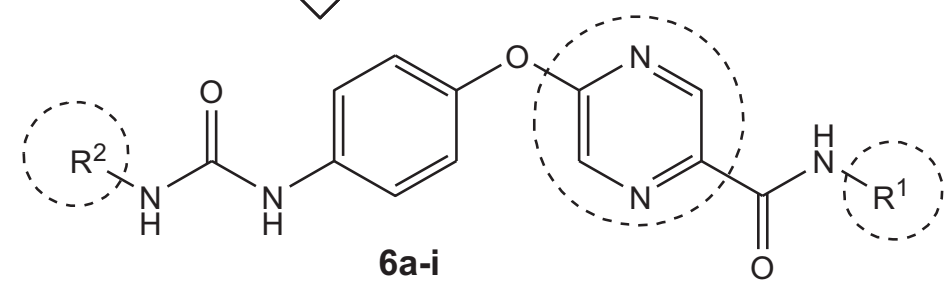

6a-i

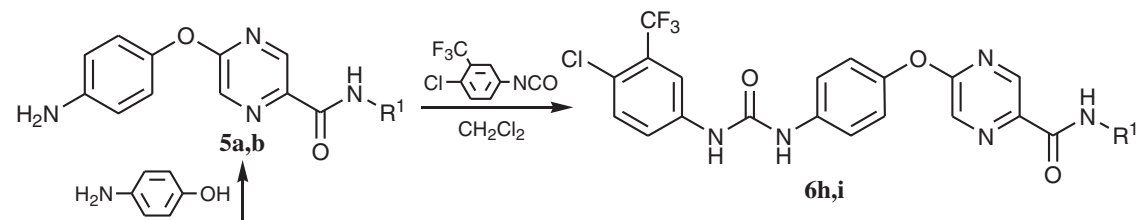
$\mathrm{K}_{2} \mathrm{CO}_{3}, \mathrm{KO}_{t} \mathrm{Bu}, \mathrm{DMF}$

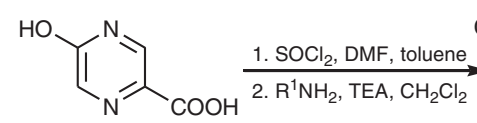<smiles>NC=C(N)Cl</smiles><smiles>NC(=O)c1cncnn1</smiles><smiles>Nc1ccc(O)c(C(F)(F)F)c1</smiles>
2<smiles>[R]NC(=O)Nc1ccc(O)cc1</smiles>
$\underset{\mathrm{DMF}}{\stackrel{\mathrm{K}_{2} \mathrm{CO}_{3}}{\longrightarrow}}$<smiles>[R]NC(=O)Nc1ccc(Oc2cnc(C(=O)N[R])cn2)cc1</smiles>
6a-i $\mathrm{R}^{1}=\mathrm{CH}_{3} \sim \mathrm{OH}_{1} \bigcirc \mathrm{CO}$ $\mathrm{Btc}=\mathrm{R}^{2}=$

Scheme 1 Synthetic pathways of intermediates 2-5 and target compounds 6a-i

Table 1 Properties of pyrazine sorafenib analogs 6a-i: the Lipinski's and Gelovani's parameters

\begin{tabular}{|c|c|c|c|c|c|c|c|c|c|}
\hline Compounds & Molecular formula & $\begin{array}{l}\text { Number of } \\
\text { atoms }\end{array}$ & MW & $\log P$ & $\begin{array}{l}\text { H-bond } \\
\text { donor }\end{array}$ & $\begin{array}{l}\text { H-bond } \\
\text { acceptor }\end{array}$ & $\begin{array}{l}\text { Lipinski } \\
\text { score }^{\mathrm{a}}\end{array}$ & $\mathrm{MR} \mathrm{cm}^{3} / \mathrm{mol}$ & PSA $\left(\AA^{2}\right)$ \\
\hline $6 \mathbf{a}$ & $\mathrm{C}_{22} \mathrm{H}_{27} \mathrm{~N}_{5} \mathrm{O}_{3}$ & 57 & 409.48 & 2.93 & 3 & 4 & 4 & 113.164 & 105.24 \\
\hline $6 \mathbf{b}$ & $\mathrm{C}_{19} \mathrm{H}_{16} \mathrm{BrN}_{5} \mathrm{O}_{3}$ & 44 & 442.27 & 3.01 & 3 & 4 & 4 & 109.519 & 105.24 \\
\hline $6 c$ & $\mathrm{C}_{23} \mathrm{H}_{22} \mathrm{BrN}_{5} \mathrm{O}_{3}$ & 54 & 496.36 & 3.62 & 3 & 4 & 4 & 125.932 & 105.24 \\
\hline 6d & $\mathrm{C}_{20} \mathrm{H}_{15} \mathrm{ClF}_{3} \mathrm{~N}_{5} \mathrm{O}_{3}$ & 47 & 465.81 & 3.72 & 3 & 4 & 4 & 112.675 & 105.24 \\
\hline $6 e$ & $\mathrm{C}_{21} \mathrm{H}_{17} \mathrm{ClF}_{3} \mathrm{~N}_{5} \mathrm{O}_{4}$ & 51 & 495.84 & 3.03 & 4 & 5 & 4 & 118.967 & 125.47 \\
\hline $6 \mathbf{f}$ & $\mathrm{C}_{26} \mathrm{H}_{19} \mathrm{ClF}_{3} \mathrm{~N}_{5} \mathrm{O}_{4}$ & 58 & 557.91 & 5.60 & 3 & 5 & 3 & 138.638 & 114.47 \\
\hline $6 \mathrm{~g}$ & $\mathrm{C}_{26} \mathrm{H}_{15} \mathrm{Cl}_{2} \mathrm{~F}_{6} \mathrm{~N}_{5} \mathrm{O}_{3}$ & 57 & 630.33 & 7.22 & 3 & 4 & 3 & 145.012 & 105.24 \\
\hline $6 h$ & $\mathrm{C}_{24} \mathrm{H}_{21} \mathrm{ClF}_{3} \mathrm{~N}_{5} \mathrm{O}_{3}$ & 57 & 519.90 & 5.07 & 3 & 4 & $2^{\mathrm{b}}$ & 129.088 & 105.24 \\
\hline $6 \mathbf{i}$ & $\mathrm{C}_{25} \mathrm{H}_{23} \mathrm{ClF}_{3} \mathrm{~N}_{5} \mathrm{O}_{3}$ & 60 & 533.93 & 5.52 & 3 & 4 & 3 & 133.689 & 105.24 \\
\hline
\end{tabular}

Calculated with Chemicalize.org program

${ }^{\text {a }}$ Out of four

${ }^{\mathrm{b}}$ Minimal aberrations of two rules; $M R$ molecular refractivity, PSA polar surface area 
4-aminophenol according to our slightly modified procedure (Butula et al., 1978). On the other hand, amides 4 were obtained by the reaction of the corresponding amine [methylamine, ethanolamine, cyclopentylamine, cyclohexylamine, $O$ benzylhydroxylamine, or 4-chloro-3-(trifluoromethyl)aniline] with 5-chloropyrazine-2-carboxylic chloride, prepared from 5-hydroxy-pyrazine-2-carboxylic acid and thionyl chloride. The amidation step was performed at room temperature in the presence of triethylamine (TEA) as $\mathrm{HCl}$ acceptor.

In the second strategy, leading to sorafenib pyrazine analogs 6, ether functionality was introduced in the molecule before the urea moiety. Amides 4 were first converted to 5-(4-aminophenoxy)- $N$-alkylpyrazine-2-carboxamides $\mathbf{5}$. Ether bond formation was performed by the reaction of precursors 4 with $p$-aminophenol in the presence of $\mathrm{K}_{2} \mathrm{CO}_{3}$ and potassium $t$-butoxyde. Under the basic conditions employed, the alkoxyde derived from 4-aminophenol was a stronger nucleophile than the amino group; therefore the main product was the ether, rather than the secondary amine. The last step in the preparation of products 6 involved urea formation between the amino group of compounds 5 and 4-chloro-3-(fluoromethyl)phenyl isocyanate. Urea bond formation was performed at room temperature, using a slight excess of isocyanate.

Preparation of compounds $\mathbf{3 b}$ and $\mathbf{3} \mathbf{c}$ has been previously published (Winum et al., 2012; Yang et al., 2013), but their analytical and spectral data were not given. Compound $\mathbf{4 a}$ is commercially available and all other compounds are new compounds. They are isolated and fully characterized by the usual spectroscopic methods [infrared (IR), ${ }^{1} \mathrm{H}-,{ }^{13} \mathrm{C}$ nuclear magnetic resonance (NMR), and MS] and elemental analyses. Spectral data are consistent with the proposed structures and are given in short in the Experimental section and in detail in Supporting information. As could be expected, the signals of carbon atoms bearing three fluoro atoms and aromatic carbon atoms adjacent to $\mathrm{CF}_{3}$ group split in ${ }^{13} \mathrm{C}$ NMR spectra and appeared as quartets.

\section{Biological evaluation}

\section{Cytostatic activity}

Eight human tumor cell lines derived from seven human cancer types: acute lymphoblastic leukemia (CEM, Molt4/ C8), cervical carcinoma (HeLa), lung adenocarcinoma (A549), hepatocellular carcinoma (HepG2), metastatic colorectal adenocarcinoma (SW620), breast carcinoma (MCF-7), murine cancer cell line (L1210), and normal human diploid fibroblasts (WI-38) derived from embryonic lung tissue were used in antiproliferative evaluation assay. The results of in vitro screening of sorafenib analogs $6 \mathbf{a}-\mathbf{i}$ against selected cell lines are presented in Table 2. The strongest cytostatic activity was exerted by 5-/4-[3-(4-chloro-3-trifluoromethyl-phenyl)-ureido]phenoxy/-pyrazine-2-carboxylic acid (4-chloro-3-trifluoromethylphenyl)-amide (6g), which showed comparable or even more potent activity than sorafenib $\left(\mathrm{IC}_{50} 0.9-7.5\right.$ $\mu \mathrm{M}) .6 \mathbf{6}, \mathbf{6 f}$, and $\mathbf{6 i}$ were also very potent against all tested cell lines, including WI-38, like sorafenib itself. The common structural feature for these compounds is 4-chloro-3trifluoromethylphenyl moiety in urea part. The most active compound 6g have 4-chloro-3-trifluoromethylphenyl residue on both wings of the molecule, e.g., both in urea and amide region. This compound was also the most liphophilic, suggesting that lipohilicity is important for anticancer activity.

Two compounds, namely, 5-/4-[3-(4-bromophenyl)ureido]-phenyloxy/-pyrazine-2-carboxylic acid cyclopentylamide (6c) and 5-/4-[3-(4-chloro-3-trifluoromethylphenyl)-ureido]-phenoxy/-pyrazine-2-carboxylic acid cyclopentylamide $(\mathbf{6 h})$, exerted cytostatic activities $\left(\mathrm{IC}_{50} 0.6-0.9\right.$ $\mu \mathrm{M})$ that significantly surpassed the effects observed with sorafenib in three cancer cell lines (HepG2, HeLa, A549). Similar to sorafenib, compound $\mathbf{6 h}$ proved to be cytotoxic to normal human fibroblast cells, whereas compound $\mathbf{6 c}$ did not diminish proliferative capacity of these cells $\left(\mathrm{IC}_{50}>100\right.$ $\mu \mathrm{M})$. Compounds $6 \mathbf{a}$ and $\mathbf{6 b}$ showed also high cytostatic activity against the same cancer cell lines (6a: HeLa and HepG2; 6b: HeLa and A549). All these compounds have either cyclopentyl residue in amide part or bromophenyl group in urea part or both these structural features (compound 6c).

All compounds exerted cytostatic activity against HeLa cell line. However, most of the compounds were active toward WI-38 as well and showed comparable activity to that observed for sorafenib. Compounds $\mathbf{6 b}-\mathbf{d}$ were exceptions: 6b and 6d showed low toxicity toward fibroblast WI-38 ( IC $_{50} 58.1$ and $21.9 \mu \mathrm{M}$, respectively) and $6 \mathbf{c}$ no toxicity. It is interesting to note that compound $\mathbf{6 d}$, the most similar compound to sorafenib, with the same urea and amide part, but different heterocycle, showed much higher selectivity than sorafenib itself: it was very active against three cancer cell lines (HepG2, HeLa, and A549 again) and practically inactive against other five. It also showed lower toxicity to fibroblast WI-38 than sorafenib. The observed selectivity could be fully attributed to pyrazine moiety.

In conclusion, all the tested compounds showed strong antiproliferative activity in the low micromolar range or high selectivity against the selected cancer cell lines. Compound 6c may be considered as the most promising pyrazine sorafenib analog, due to more potent anticancer activity against three cancer cell lines, reduced toxicity in comparison with sorafenib, and full agreement with the Lipinski's and Gelovani's rules for prospective small molecular drugs. It could be used as a scaffold for 


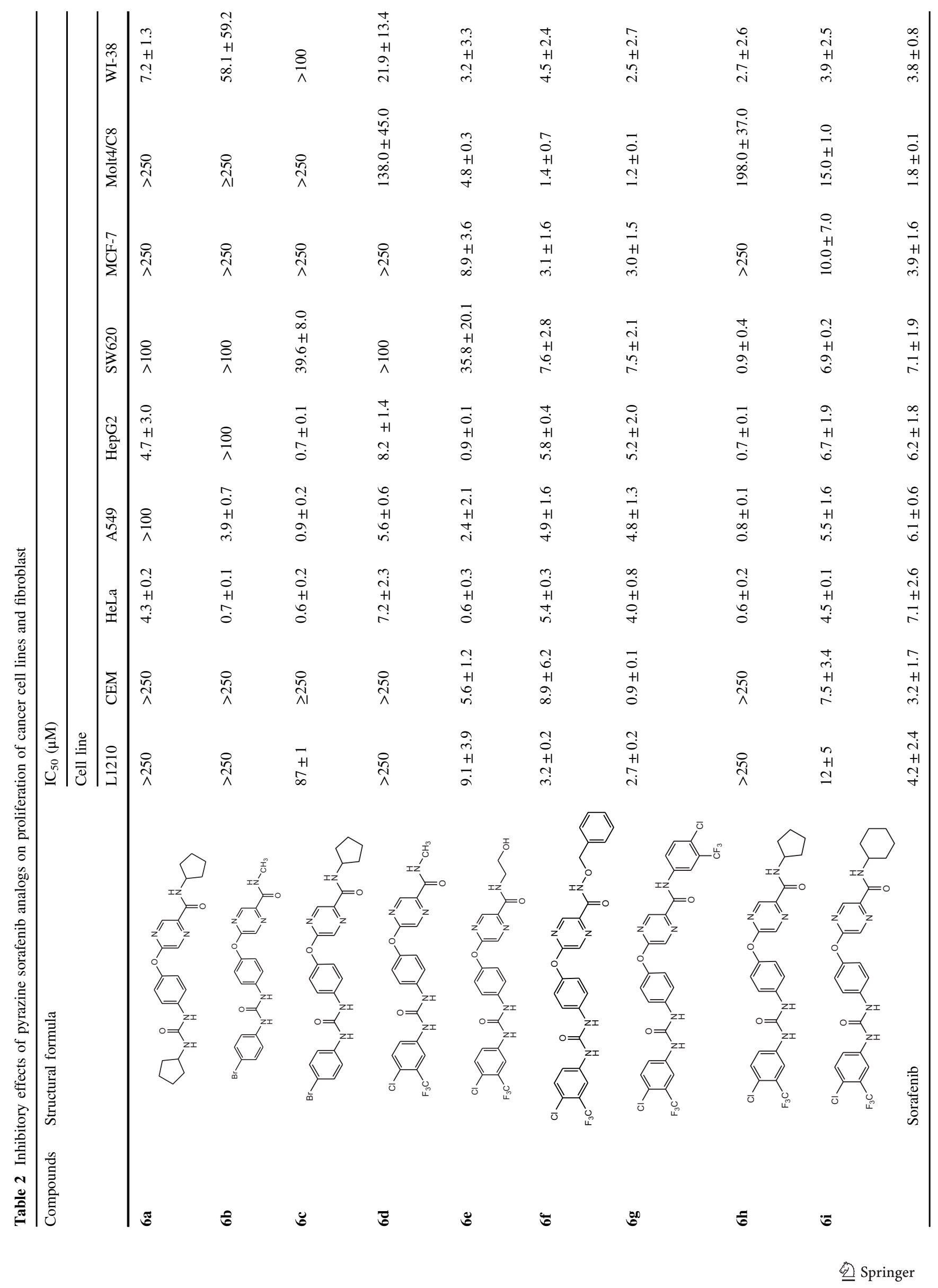




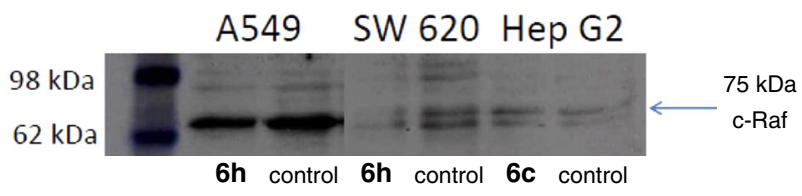

Fig. 2 Representative Western blots for expression of phospho-c-Raf (Ser259), 75kDa in cancer cell lines treated with compounds $\mathbf{6 h}$ and $\mathbf{6 c}$ after 24h. Downregulation of phospho-c-Raf is visible in A549 and SW 620 cells treated with $\mathbf{6 h}$

development of new, more effective, and safer drugs for treating hepatocellular, lung, and cervical carcinoma.

\section{c-Raf kinase inhibition}

In vitro antiproliferative screening identified two compounds, $\mathbf{6 h}$ and $\mathbf{6 c}$, with potent cytostatic activity that surpassed the effects observed with sorafenib in three cancer cell lines (HepG2, HeLa, A549). To investigate if compounds $6 \mathbf{c}$ and $\mathbf{6 h}$ share the common cytostatic mechanism with sorafenib, we analyzed the effects of these compounds on the activity of c-Raf kinase, known sorafenib target, using Western blot method with specific phospho-c-Raf (Ser259) antibody.

The method detects endogenous levels of c-Raf only when phosphorylated at Ser259, indicating its activation. Western blot analysis clearly showed marked downregulation of phospho-c-Raf in A549 and SW620 cell lines treated with $\mathbf{6 h}$ at its $\mathrm{IC}_{50}$ value for $48 \mathrm{~h}$, which suggests that $\mathbf{6 h}$ acts as c-Raf inhibitor. On contrary, compound $\mathbf{6 c}$ did not elicit significant change in the expression level of phospho-c-Raf in HepG2 cells, which suggests that other mechanism than c-Raf inhibition may account for its observed cytostatic activity in hepatocellular carcinoma cells. This striking difference between compounds $\mathbf{6 h}$ and 6c in the mechanism underlying their antiproliferative effects as well as opposing cytotoxic effects in normal human fibroblasts could be attributed to their structural differences. Both compounds possess the same cyclopentyl substituent in amide region and differ only in the urea part of the molecules: $\mathbf{6 h}$ has trifluoromethyl and chlorosubstituted benzene ring as sorafenib, while $\mathbf{6 c}$ is a bromophenyl derivative. The results suggest that the urea part of the molecule is essential for the interaction between $\mathbf{6 h}$ and c-Raf (Fig. 2).

\section{Experimental}

\section{Chemistry}

Melting points were determined on a Stuart Melting Point Apparatus SMP3 and were uncorrected. IR spectra were recorded on a Fourier transform-infrared spectroscopy
Perkin Elmer Paragon 500 spectrometer (Perkin-Elmer, UK). ${ }^{1} \mathrm{H}$ and ${ }^{13} \mathrm{C}$ NMR spectra were recorded on a Bruker AV-600 spectrometer (Bruker, USA) operating at 300 and $600 \mathrm{MHz}$ for ${ }^{1} \mathrm{H}$ and 75 and $150 \mathrm{MHz}$ for ${ }^{13} \mathrm{C}$ nuclei. Samples were measured in dimethyl sulfoxide (DMSO)- $d_{6}$ solutions at $20^{\circ} \mathrm{C}$ in 5 -mm NMR tubes. Chemical shifts $(\delta)$ in $\mathrm{ppm}$ were referred to tetramethylsilane. Mass spectra were taken on a high-performance liquid chromatographymass spectrometry (HPLC, Agilent Technologies 1200 Series; MS, Agilent Technologies 6410 Triple Quad). Elemental analyses were performed on a CHNS LECO analyzer (LECO Corporation, USA). Analyses indicated by the symbols of the elements were within $\pm 0.4 \%$ of the theoretical values. All compounds are routinely checked by thinlayer chromatography (TLC) with Merck silica gel 60F-254 glass plates using the following solvent systems: cyclohexane/ethyl acetate/methanol 3:1:0.5, cyclohexane/ ethyl acetate $3: 1,1: 1$, and 1:2, ethyl acetate/methanol 2:0.1, dichloromethane/methanol/cyclohexane/ethyl acetate 9:1:5:5, dichloromethane/methanol 9.5:0.5, ethyl acetate/ petrolether/methanol 2:2:0.1 and 3:1:0.1, petrolether/ethyl acetate $1: 1$, and ethyl acetate. Spots were visualized by short-wave UV light and iodine vapour. Column chromatography was performed on silica gel $(0.063-0.200 \mathrm{~mm})$, with the same eluents used in TLC. $1 H$-benzo $[d][1,2,3]$ triazole $(\mathrm{BtH}), 5$-hydroxy-pyrazine-2-carboxylic acid, methylamine hydrochloride, cyclopentylamine, cyclohexylamine, ethanolamine, 4-bromoaniline, 4-chloro-3-(fluoromethyl)aniline, $O$-benzylhydroxylamine hydrochloride, and potassium $t$-butoxide $\left(\mathrm{KO}_{t} \mathrm{Bu}\right)$ were purchased from Aldrich (Germany), 4-aminophenol and 4-chloro-3-(fluoromethyl)phenyl isocyanate from Acros Organics (Belgium), triphosgene from Alpha Aesar (USA), and TEA from Sigma Chemical Co. (USA). 1-benzotriazole carboxylic acid chloride $(\mathrm{BtcCl}, \mathbf{1})$ was prepared from $\mathrm{BtH}$ and triphosgene following our published procedure (Butula et al., 1977; Butula and Jadrijević-Mladar Takač, 2000).

$\mathrm{N}$-(4-hydroxyphenyl)-1H-benzo[d][1,2,3]triazole-1-carboxamide (2) A solution of $1.086 \mathrm{~g}(6.0 \mathrm{mmol}) \mathrm{BtcCl}(\mathbf{1})$ in $25 \mathrm{ml}$ of dry dioxane was added dropwise to a solution of equimolar amount of $p$-aminophenol $(0.655 \mathrm{~g})$ and TEA $(0.606 \mathrm{~g})$ in $30 \mathrm{ml}$ of dry dioxane. Reaction mixture was stirred $30 \mathrm{~min}$ at room temperature. TEA $\times \mathrm{HCl}$ was filtered off and the mother liquor was evaporated under reduced pressure. After purification by column chromatography (mobile phase dichloromethane/methanol 9.5:0.5) and crystallization from toluene and acetone/water, $0.915 \mathrm{~g}$ (60\%) of 2 was obtained; mp. $240{ }^{\circ} \mathrm{C}$ (decomp.); IR (KBr): $\nu_{\max } 3456,3252,1726,1544,1616,1544,1618,1488$, 1446, 1370, 1236, 1212, 1060, 926, 825, 146, $583 \mathrm{~cm}^{-1} ;{ }^{1} \mathrm{H}$ NMR (DMSO, $300 \mathrm{MHz}$ ): $\delta=10.89$ (s, 1H, 1), 9.41 (s, 1H, 8), 8.24 (d, $J=9.12,2 \mathrm{H}$, arom.), 7.76-7.73 (m, 1H, arom.), 
7.60-7.57 (m, 3H, arom.), 6.83-6.81 (m, 2H, arom.); ${ }^{13} \mathrm{C}$ NMR (DMSO, $75 \mathrm{MHz}$ ): $\delta=154.61$ (9), 146.99 (2), 145.50 (15), 131.47 (10), 129.94 (13), 128.40 (5), 125.62 (12), 123.19 (4, 6), 119.18 (14), 115.18 (3, 7), 113.69 (11); ESIMS $m / z$ (pos): 277.1, $\mathrm{C}_{13} \mathrm{H}_{10} \mathrm{~N}_{4} \mathrm{NaO}_{2}$ (calcd. 277.2).

General procedure for preparation of 4-hydroxyphenyl ureas 3a-c Method A: A suspension of $0.280 \mathrm{~g}$ (1.1 mmol) benzotriazole-1-carboxylic acid (4-hydroxyphenyl)amide (2) and corresponding amine $(2.2 \mathrm{mmol})$ in $20 \mathrm{ml}$ of ethanol was heated $1 \mathrm{~h}$ at $50^{\circ} \mathrm{C}$. The reaction mixture was evaporated under reduced pressure. Method B: A suspension of $0.280 \mathrm{~g}(1.1 \mathrm{mmol})$ benzotriazole-1-carboxylic acid (4-hydroxyphenyl)-amide (2) and corresponding amine (2.2 $\mathrm{mmol}$ ) in $25 \mathrm{ml}$ of DMF was heated $3 \mathrm{~h}$ at $115^{\circ} \mathrm{C}$. The reaction mixture was evaporated under reduced pressure. Products 3a-c were purified by column chromatography or by crystallization.

1-cyclopentyl-3-(4-hydroxyphenyl)urea (3a) This compound was prepared by the reaction of $0.280 \mathrm{~g}(1.1 \mathrm{mmol})$ amide 2 and $0.113 \mathrm{~g}(2.2 \mathrm{mmol})$ cyclopentylamine. After purification by column chromatography (mobile phase petrolether/etyl acetate $1: 1), 0.208 \mathrm{~g}(86 \%)$ of a white solid was obtained; mp. $175-177^{\circ} \mathrm{C}$; IR (KBr): $\nu_{\max } 3243,2960$, 2870, 1608, 1574, 1517, 1442, 1309, 1259, 1220, 1163, 835, 805, 727, 667, $523 \mathrm{~cm}^{-1}$; ${ }^{1} \mathrm{H}$ NMR (DMSO, 300 $\mathrm{MHz}): \delta=8.88(\mathrm{~s}, 1 \mathrm{H}, 1), 7.86(\mathrm{~s}, 1 \mathrm{H}, 8), 7.13-7.11(\mathrm{~m}$, 2H, arom.), 6.63-6.60 (m, 2H, arom.), 5.94 (d, J=7.20, $1 \mathrm{H}, 10), 3.93-3.88(\mathrm{~m}, 1 \mathrm{H}, 11), 1.84-1.78$ (m, 2H, 12), 1.64-1.59 (m, 2H, 15), 1.55-1.50 (m, 2H, 13), 1.36-1.30 (m, 2H, 12); ${ }^{13} \mathrm{C}$ NMR (DMSO, $\left.75 \mathrm{MHz}\right): \delta=155.11$ (9), 151.79 (2), 132.07 (5), $119.60(4,6), 115.02(3,7), 50.82$ (11), 32.86 (12, 15), 23.10 (13, 14); ESIMS m/z (pos): 221.1, $\mathrm{C}_{12} \mathrm{H}_{17} \mathrm{~N}_{2} \mathrm{O}_{2}$ (calcd. 221.3); Anal. Calcd for $\mathrm{C}_{12} \mathrm{H}_{16} \mathrm{~N}_{2} \mathrm{O}_{2}$ : C, 65.43; H, 7.32; N, 12.72. Found: C, 65.16, $\mathrm{H}, 6.96 ; \mathrm{N}, 12.35$.

1-(4-bromophenyl)-3-(4-hydroxyphenyl)urea (3b) This compound was prepared by the reaction of $0.280 \mathrm{~g}$ (1.1 mmol) amide 2 and $0.378 \mathrm{~g}(2.2 \mathrm{mmol})$ 4-bromoaniline. After crystallization from dichloromethane, $0.301 \mathrm{~g}(89 \%)$ of a white solid was obtained; mp. $244-245^{\circ} \mathrm{C}$ (decomp.); IR (KBr): $\nu_{\max } 3302,1637,1590,1565,1509,1488,1464$, 1393, 1302, 1224, 1102, 1071, 1010, 853, 824, 793, 649 $\mathrm{cm}^{-1}$; ${ }^{1} \mathrm{H}$ NMR (DMSO, $300 \mathrm{MHz}$ ): $\delta=9.05(\mathrm{~s}, 1 \mathrm{H}, 1)$, 8.65 (s, 1H, 8), 8.33 (s, 1H, 10), 7.41 (s, 4H, 12, 13, 15, 16), 7.23-7.18 (m, 2H, 3, 7), 6.71-6.66 (m, 2H, 4, 6); ${ }^{13} \mathrm{C} \mathrm{NMR}$ (DMSO, $75 \mathrm{MHz}$ ): $\delta=152.68$ (2), 152.61 (9), 139.42 (11), 131.39 (12, 16), 130.82 (5), 120.58 (4, 6), 119.89 (13, 15), 115.16 (3, 7), 112.77 (14); ESIMS m/z (pos): 309.1, $\mathrm{C}_{13} \mathrm{H}_{12}{ }^{81} \mathrm{BrN}_{2} \mathrm{O}_{2}$ (calcd. 309.1); 307.2, $\mathrm{C}_{13} \mathrm{H}_{12}{ }^{79} \mathrm{BrN}_{2} \mathrm{O}_{2}$ (calcd. 307.1); Anal. Calcd for $\mathrm{C}_{13} \mathrm{H}_{11} \mathrm{BrN}_{2} \mathrm{O}_{2}$ : C, 50.85; $\mathrm{H}$, 3.61 ; N, 9.12. Found: C, 50.65, H, 3.99; N, 9.02. 1-(4-chloro-3-(trifluoromethyl)phenyl)-3-(4-hydroxyphenyl) urea (3c) This compound was prepared by the reaction of $0.280 \mathrm{~g}$ ( $1.1 \mathrm{mmol})$ amide 2 and $0.430 \mathrm{~g}(2.2 \mathrm{mmol}) 4$ chloro-3-(trifluoromethyl)aniline. After purification by column chromatography (mobile phase dichloromethane/ methanol 9.5:0.5), $0.109 \mathrm{~g}(30 \%)$ of a white solid was obtained; mp. $210-215^{\circ} \mathrm{C}$; IR (KBr): $\nu_{\max } 3305,3112$, 2930, 1673, 1625, 1591, 1560, 1482, 1436, 1328, 1268, 1185, 1147, 1126, 832, $684 \mathrm{~cm}^{-1} ;{ }^{1} \mathrm{H}$ NMR (DMSO, 300 $\mathrm{MHz}): \delta=9.09$ (s, 1H, 1), 9.00 (s, 1H, 8), 8.46 (s, 1H, 10), $8.09(\mathrm{~d}, J=2.13,1 \mathrm{H}, 16), 7.64-7.56(\mathrm{~m}, 2 \mathrm{H}, 12,13)$, 7.24-7.19 (m, 2H, 3, 7), 6.72-6.67 (m, 2H, 4, 6); ${ }^{13} \mathrm{C} \mathrm{NMR}$ (DMSO, $75 \mathrm{MHz}$ ): $\delta=152.95$ (1), 152.60 (9), 139.66 (11), 131.88 (13), 130.45 (5), 126.93-126.32 (q, $J=30.57,15)$, 125.53-120.10 (q, $J=272.90,17), 122.77$ (12), 121.84 (14), $120.99(4,6), 116.57-116.45$ (q, $J=5.72,16), 115.17(3,7)$; ESIMS $m / z$ (pos): 330.7, $\mathrm{C}_{14} \mathrm{H}_{10} \mathrm{ClF}_{3} \mathrm{~N}_{2} \mathrm{O}_{2}$ (calcd. 330.7); Anal. Calcd for $\mathrm{C}_{14} \mathrm{H}_{10} \mathrm{ClF}_{3} \mathrm{~N}_{2} \mathrm{O}_{2}$ : C, 50.85; H, 3.05; N, 8.47. Found: C, 50.49, H, 3.22; N, 8.88.

General procedure for preparation of 5-chloro-pyrazine-2carboxamides $\mathbf{4 a}-\mathbf{f} \quad$ A suspension of $0.308 \mathrm{~g}$ (2.2 mmol) 5hydroxy-2-pyrazine carboxylic acid, $1.666 \mathrm{~g}$ (14.0 mmol) thionyl chloride, and 2 drops of DMF in $30 \mathrm{ml}$ of dry toluene was heated at $100{ }^{\circ} \mathrm{C} 70 \mathrm{~min}$. The reaction mixture was cooled, filtrated, and evaporated under reduced pressure. The red oil residue was dissolved in $10 \mathrm{ml}$ of dry dichloromethane and added dropwise to a cold solution $\left(0{ }^{\circ} \mathrm{C}\right)$ of corresponding amine $(1.7 \mathrm{mmol})$ and $0.404 \mathrm{~g}$ (4.0 mmol) TEA in $20 \mathrm{ml}$ of dry dichloromethane. The reaction mixture was stirred $30 \mathrm{~min}$ and evaporated under reduced pressure. Products $\mathbf{4 a}-\mathbf{f}$ were purified by column chromatography or by crystallization.

5-chloro-N-methylpyrazine-2-carboxamide (4a) This compound was prepared by the reaction of $0.308 \mathrm{~g}(2.2$ mmol) 5-hydroxy-2-pyrazine carboxylic acid, $1.666 \mathrm{~g}$ (14.0 mmol) thionyl chloride, $0.144 \mathrm{~g}(1.7 \mathrm{mmol})$ methylamine hydrochloride, and $0.606 \mathrm{~g}(6.0 \mathrm{mmol})$ TEA. After purification by column chromatography (mobile phase ethyl acetate/petrolether/methanol 3:1:0.1), $0.198 \mathrm{~g}(68 \%)$ of a white solid was obtained; mp. $153-156^{\circ} \mathrm{C}$; IR (KBr): $\nu_{\max }$ 3369, 3078, 2944, 1660, 1573, 1536, 1517, 1456, 1412, 1278, 1181, 1126, 1032, 1003, 932, 852, 664, 632, 522, $491 \mathrm{~cm}^{-1}$; ${ }^{1} \mathrm{H}$ NMR (DMSO, $\left.300 \mathrm{MHz}\right): \delta=8.99(\mathrm{~d}, J=$ $1.32,1 \mathrm{H}, 5), 8.89-8.86\left(\mathrm{~m}, 2 \mathrm{H}, 3,1^{\prime}\right), 2.83$ (d, 3H, $J=4.83$, $2^{\prime}$ ); ${ }^{13} \mathrm{C}$ NMR (DMSO, $75 \mathrm{MHz}$ ): $\delta=162.40$ (1), 150.54 (4), 143.59 (2), 143.17 (5), 143.00 (3), 25.98 (2'); ESIMS $m / z$ (pos): 172.1, $\mathrm{C}_{6} \mathrm{H}_{7} \mathrm{ClN}_{3} \mathrm{O}$ (calcd. 171.6); Anal. Calcd for $\mathrm{C}_{6} \mathrm{H}_{6} \mathrm{ClN}_{3} \mathrm{O}$ : C, 42.00; H, 3.52; N, 24.49. Found: C, 42.33, H, 3.00; N, 24.11.

N-(2-hydroxyethyl)-5-chloropyrazine-2-carboxamide (4b) This compound was prepared by the reaction of $0.308 \mathrm{~g}$ 
(2.2 mmol) 5-hydroxy-2-pyrazine carboxylic acid, $1.666 \mathrm{~g}$ (14.0 mmol) thionyl chloride, and $0.267 \mathrm{~g}(4.3 \mathrm{mmol})$ ethanolamine. After purification by column chromatography (mobile phase ethyl acetate/petrolether/methanol 3:1:0.1), $0.218 \mathrm{~g}(59 \%)$ of a white solid was obtained; mp. $94-96^{\circ} \mathrm{C}$; IR (KBr): $\nu_{\max } 3356,3079,2942,2879,1665,1573,1529$, 1459, 1439, 1365, 1302, 1278, 1210, 1182, 1127, 1055, 1031, 872, 794, 742, 692, $519 \mathrm{~cm}^{-1} ;{ }^{1} \mathrm{H}$ NMR (DMSO, 300 $\mathrm{MHz}): \delta=9.00(\mathrm{~d}, J=1.32,1 \mathrm{H}, 5), 8.87(\mathrm{~d}, J=1.32,1 \mathrm{H}$, 3), $8.76\left(\mathrm{t}, J=4.63,1 \mathrm{H}, 1^{\prime}\right), 4.77\left(\mathrm{t}, J=5.51,1 \mathrm{H}, 4^{\prime}\right), 3.54$ $\left(\mathrm{q}, J=5.90,2 \mathrm{H}, 3^{\prime}\right), 3.39\left(\mathrm{q}, J=5.96,2 \mathrm{H}, 2^{\prime}\right) ;{ }^{13} \mathrm{C} \mathrm{NMR}$ (DMSO, $75 \mathrm{MHz}$ ): $\delta=161.97$ (1), 150.64 (4), 143.46 (2), 143.27 (5), 143.00 (3), $59.41\left(3^{\prime}\right), 41.67$ (2'); ESIMS $\mathrm{m} / \mathrm{z}$ (pos): 224.2, $\mathrm{C}_{7} \mathrm{H}_{9} \mathrm{ClN}_{3} \mathrm{NaO}_{2}$ (calcd. 224.6); 202.2, $\mathrm{C}_{7} \mathrm{H}_{9} \mathrm{ClN}_{3} \mathrm{O}_{2}$ (calcd. 201.6); Anal. Calcd for $\mathrm{C}_{7} \mathrm{H}_{8} \mathrm{ClN}_{3} \mathrm{O}_{2}$ : C, 41.70; H, 4.00; N, 20.84. Found: C, 41.99, H, 3.68; N, 20.61 .

N-cyclopentyl-5-chloropyrazine-2-carboxamide (4c) This compound was prepared by the reaction of $0.308 \mathrm{~g}(2.2$ mmol) 5-hydroxy-2-pyrazine carboxylic acid, $1.666 \mathrm{~g}$ (14.0 mmol) thionyl chloride, $0.145 \mathrm{~g}(1.7 \mathrm{mmol})$ cyclopentylamine, and $0.404 \mathrm{~g}$ (4.0 mmol) TEA. After purification by column chromatography (mobile phase dichloromethane/ methanol 9.5:0.5), $0.384 \mathrm{~g}(89 \%)$ of a white solid was obtained; mp. $107-108{ }^{\circ} \mathrm{C}$; IR (KBr): $\nu_{\max } 3373,3079$, 2951, 2870, 1660, 1571, 1528, 1466, 1295, 1176, 1161, 1125, 1027, 927, 632, 526, $492 \mathrm{~cm}^{-1} ;{ }^{1} \mathrm{H}$ NMR (DMSO, $300 \mathrm{MHz}): \delta=8.97(\mathrm{~d}, J=1.32,1 \mathrm{H}, 5), 8.84(\mathrm{~d}, J=1.52$, $1 \mathrm{H}, 3), 8.79\left(\mathrm{~d}, J=7.56,1 \mathrm{H}, 1^{\prime}\right), 4.29-4.23\left(\mathrm{~m}, 1 \mathrm{H}, 2^{\prime}\right)$, 1.91-1.86 (m, 2H, 3'), 1.72-1.67 (m, 2H, 6'), 1.63-1.51 (m, $\left.4 \mathrm{H}, 4^{\prime}, 5^{\prime}\right) ;{ }^{13} \mathrm{C} \mathrm{NMR}$ (DMSO, $\left.75 \mathrm{MHz}\right): \delta=161.56(1)$, 150.45 (4), 143.80 (2), 143.37 (5), 142.84 (3), 50.68 (2'), $31.85\left(3^{\prime}, 6^{\prime}\right), 23.52\left(4^{\prime}, 5^{\prime}\right)$; ESIMS $m / z$ (pos): 248.1, $\mathrm{C}_{10} \mathrm{H}_{12} \mathrm{ClN}_{3} \mathrm{NaO}$ (calcd. 248.7); 226.1, $\mathrm{C}_{10} \mathrm{H}_{13} \mathrm{ClN}_{3} \mathrm{O}$ (calcd. 226.7); Anal. Calcd for $\mathrm{C}_{10} \mathrm{H}_{12} \mathrm{ClN}_{3} \mathrm{O}$ : C, 53.22; $\mathrm{H}$, 5.36; N, 18.62. Found: C, 53.67, H, 5.02; N, 18.33.

N-cyclohexyl-5-chloropyrazine-2-carboxamide (4d) This compound was prepared by the reaction of $0.308 \mathrm{~g}(2.2$ mmol) 5-hydroxy-2-pyrazine carboxylic acid, $1.666 \mathrm{~g}$ (14.0 mmol) thionyl chloride, $0.169 \mathrm{~g}$ ( $1.7 \mathrm{mmol})$ cyclohexylamine, and $0.404 \mathrm{~g}(4.0 \mathrm{mmol})$ TEA. After two crystallization from ether, $0.277 \mathrm{~g}(68 \%)$ of a white solid was obtained; mp. $153-154{ }^{\circ} \mathrm{C}$; IR (KBr): $\nu_{\max } 3377,3081$, 2923, 2851, 1657, 1570, 1530, 1465, 1454, 1310, 1169, $1124,1079,1026,925,895,861,633,524,439 \mathrm{~cm}^{-1} ;{ }^{1} \mathrm{H}$ NMR (DMSO, $300 \mathrm{MHz}$ ): $\delta=8.99$ (d, $J=1.35,1 \mathrm{H}, 5)$, $8.84(\mathrm{~d}, J=1.32,1 \mathrm{H}, 3), 8.60\left(\mathrm{~d}, J=8.37,1 \mathrm{H}, 1^{\prime}\right)$, 3.85-3.74 (m, 1H, 2'), 1.81-1.69, 1.63-1.58, 1.48-1.24, 1.20-1.06 (m, 10H, $\left.33^{\prime}-7^{\prime}\right) ;{ }^{13} \mathrm{C}$ NMR (DMSO, $\left.75 \mathrm{MHz}\right): \delta$ $=160.96$ (1), 150.49 (4), 143.77 (2), 143.38 (5), 142.84 (3), $48.19\left(2^{\prime}\right), 31.93\left(3^{\prime}, 7^{\prime}\right), 25.01\left(5^{\prime}\right), 24.76\left(4^{\prime}, 6^{\prime}\right)$; ESIMS $\mathrm{m} / \mathrm{z}$ (pos): 262.1, $\mathrm{C}_{11} \mathrm{H}_{14} \mathrm{ClN}_{3} \mathrm{NaO}$ (calcd. 262.7); 240.1,
$\mathrm{C}_{11} \mathrm{H}_{15} \mathrm{ClN}_{3} \mathrm{O}$ (calcd. 240.7); Anal. Calcd for $\mathrm{C}_{11} \mathrm{H}_{14} \mathrm{ClN}_{3} \mathrm{O}$ : C, 55.12; H, 5.89; N, 17.53. Found: C, 54.96, H, 5.56; N, 17.77 .

5-chloro-N-(4-chloro-3-(trifluoromethyl)phenyl)pyrazine-2carboxamide (4e) This compound was prepared by the reaction of $0.308 \mathrm{~g}(2.2 \mathrm{mmol}) 5$-hydroxy-2-pyrazine carboxylic acid, $1.666 \mathrm{~g}(14.0 \mathrm{mmol})$ thionyl chloride, $0.332 \mathrm{~g}$ (1.7 mmol) 4-chloro-3-trifluormethylaniline, and $0.444 \mathrm{~g}$ (4.4 mmol) TEA. After crystallization with ether and recrystallization from acetone/water, $0.371 \mathrm{~g}(65 \%)$ of a white solid was obtained; mp. $149-151^{\circ} \mathrm{C}$; IR (KBr): $\nu_{\max }$ $3376,3360,3122,3083,1693,1590,1530,1423,1332$, 1262, 1251, 1180, 1136, 1116, 1023,830, $663 \mathrm{~cm}^{-1} ;{ }^{1} \mathrm{H}$ NMR (DMSO, $300 \mathrm{MHz}): \delta=11.22\left(\mathrm{~s}, 1 \mathrm{H}, 1^{\prime}\right), 9.13$ (d, $J$ $=1.20,1 \mathrm{H}, 5), 8.96(\mathrm{~d}, J=1.26,1 \mathrm{H}, 3), 8.52(\mathrm{~d}, J=2.37$, $\left.1 \mathrm{H}, 3^{\prime}\right), 8.23\left(\mathrm{dd}, J=2.31, J=6.51,1 \mathrm{H}, 7^{\prime}\right), 7.74(\mathrm{~d}, J=$ 8.79, $1 \mathrm{H}, 66^{\prime}$ ); ${ }^{13} \mathrm{C}$ NMR (DMSO, $75 \mathrm{MHz}$ ): $\delta=161.53$ (1), 151.24 (4), 144.19 (5), 143.22 (2), 142.99 (3), $137.66\left(2^{\prime}\right)$, $132.01\left(7^{\prime}\right), \quad 126.97-126.36\left(\mathrm{q}, J=30.94,4^{\prime}\right), \quad 125.43$ $\left(6^{\prime}\right), \quad 125.09\left(5^{\prime}\right), \quad 125.40-119.97 \quad\left(\mathrm{q}, \quad J=273.67,8^{\prime}\right)$, 119.56-119.45 (q, $\left.J=5.58,3^{\prime}\right)$; ESIMS $m / z \quad$ (pos): 336.2, $\mathrm{C}_{12} \mathrm{H}_{6} \mathrm{Cl}_{2} \mathrm{~F}_{3} \mathrm{~N}_{3} \mathrm{O}$ (calcd. 336.1); Anal. Calcd for $\mathrm{C}_{12} \mathrm{H}_{6} \mathrm{Cl}_{2} \mathrm{~F}_{3} \mathrm{~N}_{3} \mathrm{O}$ : C, 42.88; $\mathrm{H}, 1.80 ; \mathrm{N}, 12.50$. Found: $\mathrm{C}$, 42.65, H, 2.06; N, 12.09 .

N-(benzyloxy)-5-chloropyrazine-2-carboxamide (4f) This compound was prepared by the reaction of $0.308 \mathrm{~g}(2.2$ mmol) 5-hydroxy-2-pyrazine carboxylic acid, $1.666 \mathrm{~g} \mathrm{(14.0}$ mmol) thionyl chloride, $0.271 \mathrm{~g}(1.7 \mathrm{mmol}) O$-benzylhydroxylamine hydrochloride, and $0.444 \mathrm{~g}$ (4.4 mmol) TEA. After crystallization with ether and recrystallization from acetone/water, $0.319 \mathrm{~g}$ (67\%) of a white solid was obtained; mp. 124-125 ${ }^{\circ} \mathrm{C}$; IR (KBr): $\nu_{\max } 3246,3089,3062,3032$, 2963, 1678, 1491, 1452, 1276, 1121, 1016, 906, 751, 699, $505 \mathrm{~cm}^{-1}$; ${ }^{1} \mathrm{H}$ NMR (DMSO, $\left.300 \mathrm{MHz}\right): \delta=12.33(\mathrm{~s}, 1 \mathrm{H}$, $\left.1^{\prime}\right), 8.98(\mathrm{~d}, J=0.99,1 \mathrm{H}, 5), 8.85(\mathrm{~d}, J=1.23,1 \mathrm{H}, 3)$, 7.49-7.37 (m, 5H, 4'-8'), $4.96\left(\mathrm{~s}, 2 \mathrm{H}, 2^{\prime}\right) ;{ }^{13} \mathrm{C} \mathrm{NMR}$ (DMSO, $75 \mathrm{MHz}$ ): $\delta=160.05$ (1), 151.42 (4), 143.83 (5), 143.72 (3), 143.54 (2), $136.04\left(3^{\prime}\right), 129.30\left(5^{\prime}, 7^{\prime}\right), 128.83$ (4'), $128.78\left(6^{\prime}, 8^{\prime}\right), 77.18\left(2^{\prime}\right)$; ESIMS $m / z$ (pos): 286.2, $\mathrm{C}_{12} \mathrm{H}_{10} \mathrm{ClN}_{3} \mathrm{NaO}_{2}$ (calcd. 286.7); 264.2, $\mathrm{C}_{12} \mathrm{H}_{11} \mathrm{ClN}_{3} \mathrm{O}_{2}$ (calcd. 264.7); Anal. Calcd for $\mathrm{C}_{12} \mathrm{H}_{10} \mathrm{ClN}_{3} \mathrm{O}_{2}: \mathrm{C}, 54.66 ; \mathrm{H}$, 3.82; N, 15.94. Found: C, 54.29, H, 3.80; N, 16.03.

General procedure for preparation of 5-(4-aminophenoxy)$\mathrm{N}$-alkylpyrazine-2-carboxamides 5a, b A suspension of $0.112 \mathrm{~g}$ ( $1 \mathrm{mmol}) \mathrm{KO}_{t} \mathrm{Bu}$ and $0.11 \mathrm{~g}(1 \mathrm{mmol}) p$-aminophenol in $10 \mathrm{ml}$ of dry DMF was stirred at room temperature for $30 \mathrm{~min}$. Amide 4 (1 mmol) and $0.07 \mathrm{~g}(0.5 \mathrm{mmol})$, $\mathrm{K}_{2} \mathrm{CO}_{3}$ was added and the reaction mixture was stirred at $80^{\circ} \mathrm{C}$ for $2 \mathrm{~h}$. DMF was evaporated under reduced pressure. Products 5 were purified by column chromatography and crystallization from ether. 
5-(4-aminophenoxy)-N-cyclopentylpyrazine-2-carboxamide (5a) This compound was prepared by the reaction of $0.226 \mathrm{~g}$ ( $1 \mathrm{mmol})$ amide $4 \mathrm{c}, 0.112 \mathrm{~g}$ ( $1 \mathrm{mmol}) \mathrm{KO}_{t} \mathrm{Bu}$, and $0.11 \mathrm{~g}(1 \mathrm{mmol}) p$-aminophenol. After purification by column chromatography (mobile phase dichloromethane/ methanol 95:5) and crystallization from ether, 0.176 g (59 $\%$ ) of a white solid was obtained; mp. $142-144{ }^{\circ} \mathrm{C}$; IR (KBr): $\nu_{\max } 3458,3380,3079,3020,2938,2864,1652$, 1619, 1587, 1506, 1470, 1352, 1326, 1298, 1268, 1196, 1025, 836, $594 \mathrm{~cm}^{-1}$; ${ }^{1} \mathrm{H}$ NMR (DMSO, $300 \mathrm{MHz}$ ): $\delta=$ $8.67(\mathrm{~d}, J=1.29,1 \mathrm{H}, 3), 8.46\left(\mathrm{~d}, J=7.95,1 \mathrm{H}, 1^{\prime}\right), 8.40(\mathrm{~d}$, $J=1.29,1 \mathrm{H}, 5), 6.91-6.86(\mathrm{~m}, 2 \mathrm{H}, 7,11), 6.63-6.58(\mathrm{~m}$, $2 \mathrm{H}, 8,10), 5.09$ (s, 2H, 12), 4.31-4.19 (m, 1H, 2'), 1.93-1.81 (m, 2H, 3'), 1.75-1.65 (m, 2H, 6') 1.63-1.51 (m, $\left.4 \mathrm{H}, 4^{\prime}, 5^{\prime}\right) ;{ }^{13} \mathrm{C} \mathrm{NMR}$ (DMSO, $75 \mathrm{MHz}$ ): $\delta=162.11$ (4), 161.72 (1), 146.54 (6), 142.44 (9), 140.93 (5), 139.22 (2), 133.13 (3), 121.69 (7, 11), $114.41(8,10), 50.48\left(2^{\prime}\right), 32.02$ $\left(3^{\prime}, 6^{\prime}\right), 23.53 \quad\left(4^{\prime}, 5^{\prime}\right)$; ESIMS $m / z \quad$ (pos): 299.3, $\mathrm{C}_{16} \mathrm{H}_{19} \mathrm{~N}_{4} \mathrm{O}_{2}$ (calcd. 299.3); Anal. Calcd for $\mathrm{C}_{16} \mathrm{H}_{18} \mathrm{~N}_{4} \mathrm{O}_{2}$ : C, 64.411; H, 6.08; N, 18.78. Found: C, 64.86, H, 5.66; N, 18.53 .

5-(4-aminophenoxy)-N-cyclohexylpyrazine-2-carboxamide (5b) This compound was prepared by the reaction of $0.240 \mathrm{~g}(1 \mathrm{mmol})$ amide $4 \mathbf{d}, 0.112 \mathrm{~g}(1 \mathrm{mmol}) \mathrm{KO}_{t} \mathrm{Bu}$, and $0.11 \mathrm{~g}$ ( $1 \mathrm{mmol}) p$-aminophenol. After purification by column chromatography (mobile phase cyclohexane/ethyl acetate $1: 2)$ and crystallization from ether, $0.140 \mathrm{~g}(45 \%)$ of a white solid was obtained; mp. $167-170{ }^{\circ} \mathrm{C}$; IR (KBr): $\nu_{\max }$ 3404, 3336, 3018, 2929, 2855, 1661, 1609, 1579, 1522, 1504, 1459, 1344, 1326, 1262, 1181, 1022, 890, 834, 593, $509 \mathrm{~cm}^{-1}$; ${ }^{1} \mathrm{H}$ NMR (DMSO, $300 \mathrm{MHz}$ ): $\delta=8.67$ (d, $J=$ $1.29,1 \mathrm{H}, 3), 8.39$ (d, $J=1.32,1 \mathrm{H}, 5), 8.32$ (d, $J=8.52,1 \mathrm{H}$, $\left.1^{\prime}\right)$, 6.91-6.86 (m, 2H, 7, 11), 6.63-6.58 (m, 2H, 8, 10), $5.06(\mathrm{~s}, 2 \mathrm{H}, 12), 3.84-3.72\left(\mathrm{~m}, 1 \mathrm{H}, 2^{\prime}\right), 1.80-1.70$, $1.62-1.56,1.47-1.25,1.20-1.11\left(\mathrm{~m}, 10 \mathrm{H}, 3^{\prime}-7^{\prime}\right) ;{ }^{13} \mathrm{C} \mathrm{NMR}$ (DMSO, $75 \mathrm{MHz}$ ): $\delta=161.70$ (4), 161.47 (1), 146.48 (6), 142.45 (9), 140.92 (5), 139.17 (2), 133.09 (3), 121.60 (7, 11), $114.38(8,10), 47.85\left(2^{\prime}\right), 32.10\left(3^{\prime}, 7^{\prime}\right), 25.04\left(5^{\prime}\right)$, 24.76 (4', 6'); ESIMS $m / z$ (pos): 313.3, $\mathrm{C}_{17} \mathrm{H}_{21} \mathrm{~N}_{4} \mathrm{O}_{2}$ (calcd. 313.4); Anal. Calcd for $\mathrm{C}_{17} \mathrm{H}_{20} \mathrm{~N}_{4} \mathrm{O}_{2}$ : C, 65.37; $\mathrm{H}, 6.45 ; \mathrm{N}$, 17.94. Found: C, 65.29, H, 6.56; N, 17.61.

General procedure for preparation of pyrazine sorafenib analogs 6a-i Method A: A suspension of 5-chloro-pyrazine-2-carboxamide 4 (0.4 mmol), urea 3 (0.4 mmol), and $0.111 \mathrm{~g}(0.8 \mathrm{mmol})$ potassium carbonate in $4 \mathrm{ml}$ of dry DMF was heated at $100{ }^{\circ} \mathrm{C}$ for $3-4.5 \mathrm{~h}$. The reaction mixture was cooled to room temperature, diluted with $30 \mathrm{ml}$ ethyl acetate, and extracted three times with brine. Organic layer was dried over anhydrous sodium sulfate, filtered, and evaporated under reduced pressure. The crude products were purified by column chromatography on silica gel using petrolether/ethyl acetate/methanol (3:1:0.5, v/v) (6b), petrolether/ethyl acetate/methanol $(2: 2: 01, v / v)(\mathbf{6 d}$ and $\mathbf{6 f})$, dichloromethane/methanol $(9.5: 0.5, v / v)(\mathbf{6 g})$ or by crystallization from ether $(\mathbf{6 a}, \mathbf{6 h}-\mathbf{i})$ or methanol $(\mathbf{6 e})$. Method B: A suspension of 5-(4-aminophenoxy)- $N$-alkylpyrazine-2carboxamide $5(0.2 \mathrm{mmol})$ and $0.066 \mathrm{~g}(0.3 \mathrm{mmol}) \quad 4-$ chloro-3-(trifluoromethyl)phenylisocyanate in $3 \mathrm{ml}$ of dry dichloromethane was stirred at room temperature for $2 \mathrm{~h}$. The precipitated products $\mathbf{6 h}$ or $\mathbf{6 i}$ were filtered off and crystallized from ether.

5-[4-(3-cyclopentyl-ureido)-phenyloxy]-pyrazine-2-carbocylic acid cyclopentylamide (6a) This compound was prepared by the reaction of $0.090 \mathrm{~g}(0.4 \mathrm{mmol})$ amide $4 \mathrm{c}$ and $0.088 \mathrm{~g}(0.4 \mathrm{mmol})$ urea 3a (Method A). After crystallization from ether, $0.067 \mathrm{~g}(41 \%)$ of a white solid was obtained; mp. $235-237^{\circ} \mathrm{C}$; IR (KBr): $\nu_{\max } 3341,3070$, 3052, 2952, 2867, 1660, 1606, 1571, 1505, 1461, 1408, 1349, 1316, 1284, 1242, 1194, 1023, 897, 835, 626, 527, $502 \mathrm{~cm}^{-1}$; ${ }^{1} \mathrm{H}$ NMR (DMSO, $\left.300 \mathrm{MHz}\right): \delta=8.67(\mathrm{~d}, J=$ $1.29,1 \mathrm{H}, 3), 8.48(\mathrm{~d}, J=1.32,1 \mathrm{H}, 5), 8.43(\mathrm{~d}, J=2.88,1 \mathrm{H}$, $\left.1^{\prime}\right), 8.32$ (s, 1H, 12), 7.46-7.41 (m, 2H, 8, 10), 7.12-7.07 $(\mathrm{m}, 2 \mathrm{H}, 7,11), 6.15(\mathrm{~d}, J=7.23,1 \mathrm{H}, 14), 4.31-4.19(\mathrm{~m}$, $\left.1 \mathrm{H}, 2^{\prime}\right), 4.00-3.89(\mathrm{~m}, 1 \mathrm{H}, 15), 1.93-1.79,1.72-1.50$, 1.42-1.32 (3m, 16H, 3'-6', 16-19); ${ }^{13} \mathrm{C}$ NMR (DMSO, 75 $\mathrm{MHz}): \delta=162.01$ (4), 161.21 (1), 154.78 (13), 146.18 (6), 140.82 (5), 139.57 (2), 138.06 (9), 133.87 (3), 121.45 (8, 10), $118.70(7,11), 50.86(15), 50.48\left(2^{\prime}\right), 32.78\left(3^{\prime}, 6^{\prime}\right)$, 31.99 (16, 19), $23.49\left(4^{\prime}, 5^{\prime}\right), 23.62$ (17, 18); ESIMS $m / z$ (pos): 432.2, $\mathrm{C}_{22} \mathrm{H}_{27} \mathrm{~N}_{5} \mathrm{NaO}_{3}$ (calcd. 432.5), 410.2, $\mathrm{C}_{22} \mathrm{H}_{28} \mathrm{~N}_{5} \mathrm{O}_{3}$ (calcd. 410.5); Anal. Calcd for $\mathrm{C}_{22} \mathrm{H}_{27} \mathrm{~N}_{5} \mathrm{O}_{3}$ : C, 64.53; H, 6.65; N, 17.10. Found: C, 64.72, H, 6.60; N, 16.82 .

5-/4-[3-(4-bomophenyl)-ureido]-phenoxy/-pyrazine-2-carboxylic acid methylamide (6b) This compound was prepared by the reaction of $0.069 \mathrm{~g}(0.4 \mathrm{mmol})$ amide $4 \mathbf{a}$ and $0.123 \mathrm{~g}(0.4 \mathrm{mmol})$ urea 3b (Method A). After purification by column chromatography (mobile phase petrolether/ethyl acetate/methanol 3:1:0.5) and crystallization from ether, $0.058 \mathrm{~g}(33 \%)$ of a white solid was obtained; IR (KBr): $\nu_{\max } 3311,1662,1646,1591,1551,1505,1457,1348$, 1309, 1271, 1236, 1194, 1008, $831 \mathrm{~cm}^{-1}$; ${ }^{1} \mathrm{H}$ NMR (DMSO, $300 \mathrm{MHz}): \delta=8.85$ (s, 1H, 14), 8.80 (s, 1H, 12), 8.73-8.69 $\left(\mathrm{m}, 2 \mathrm{H}, 3,1^{\prime}\right), 8.53(\mathrm{~d}, J=1.25,1 \mathrm{H}, 5), 7.55-7.49(\mathrm{~m}, 2 \mathrm{H}$, 8, 10), 7.45 (s, 4H, 16, 17, 19, 20), 7.21-7.16 (m, 2H, 7, 11), $2.82\left(\mathrm{~d}, J=4.87,3 \mathrm{H}, 2^{\prime}\right) ;{ }^{13} \mathrm{C}$ NMR (DMSO, $75 \mathrm{MHz}$ ): $\delta=162.95$ (4), 161.21 (1), 152.45 (13), 146.98 (6), 140.66 (5), 139.58 (15), 139.11 (2), 137.01 (9), 133.63 (3), 131.50 (17, 19), $121.76(16,20), 120.13(8,10), 119.68$ (7, 11), 113.21 (18), $25.91 \quad\left(2^{\prime}\right) ; \quad$ ESIMS $m / z \quad$ (neg): 442.1, $\mathrm{C}_{19} \mathrm{H}_{15}{ }^{81} \mathrm{BrN}_{5} \mathrm{O}_{3}$ (calcd. 442.3), 440.0, $\mathrm{C}_{19} \mathrm{H}_{15}{ }^{79} \mathrm{BrN}_{5} \mathrm{O}_{3}$ (calcd. 440.3); Anal. Calcd for $\mathrm{C}_{19} \mathrm{H}_{16} \mathrm{BrN}_{5} \mathrm{O}_{3}$ : C, 51.60; $\mathrm{H}$, 3.65; N, 15.84. Found: C, 51.99, H, 3.36; N, 15.72. 
5-/4-[3-(4-bromophenyl)-ureido]-phenyloxy/-pyrazine-2carboxylic acid cyclopentylamide (6c) This compound was prepared by the reaction of $0.090 \mathrm{~g}(0.4 \mathrm{mmol})$ amide 4c and $0.123 \mathrm{~g}(0.4 \mathrm{mmol})$ urea 3b (Method A). After crystallization from ether/petrolether and recrystallization from acetone/water, $0.107 \mathrm{~g}(54 \%)$ of a white solid was obtained; mp. $238-241{ }^{\circ} \mathrm{C}$ (decomp.); IR (KBr): $\nu_{\max } 3306$, 2951, 2870, 1660, 1640, 1591, 1533, 1505, 1469, 1348, 1302, 1277, 1240, 1198, 1071, 1024, 1011, 903, 831, 657 $\mathrm{cm}^{-1} ;{ }^{1} \mathrm{H}$ NMR (DMSO, $\left.300 \mathrm{MHz}\right): \delta=8.84(\mathrm{~s}, 1 \mathrm{H}, 14)$, 8.79 (s, 1H, 12), 8.69 (d, $J=1.21,1 \mathrm{H}, 3), 8.51$ (d, $J=1.17$, $1 \mathrm{H}, 5), 8.47$ (d, $\left.J=7.93,1 \mathrm{H}, 1^{\prime}\right), 7.55-7.51$ (m, 2H, 8, 10), 7.45 (s, 4H, 16, 17, 19, 20), 7.19-7.17 (m, 2H, 7, 11), 4.28-4.22 (m, 1H, 2'), 1.91-1.87 (m, 2H, 3'), 1.73-1.67 (m, 2H, 6'), 1.61-1.51 (m, 4H, 4', 5'); ${ }^{13} \mathrm{C}$ NMR (DMSO, 75 MHz): $\delta=162.01$ (4), 161.14 (1), 152.43 (13), 147.02 (6), 140.83 (5), 139.66 (15), 139.10 (2), 136.97 (9), 133.47 (3), 131.47 (17, 19), $121.67(16,20), 120.13(8,10), 119.67$ (7, 11), 113.20 (18), $50.49\left(2^{\prime}\right), 32.00\left(3^{\prime}, 6^{\prime}\right), 23.51\left(4^{\prime}, 5^{\prime}\right)$; ESIMS $m / z$ (neg): 496.1, $\mathrm{C}_{23} \mathrm{H}_{21}{ }^{81} \mathrm{BrN}_{5} \mathrm{O}_{3}$ (calcd. 496.4), 494.1, $\mathrm{C}_{23} \mathrm{H}_{21}{ }^{79} \mathrm{BrN}_{5} \mathrm{O}_{3}$ (calcd. 494.4); Anal. Calcd for $\mathrm{C}_{23} \mathrm{H}_{22} \mathrm{BrN}_{5} \mathrm{O}_{3}$ : C, 55.65; H, 4.47; N, 14.11. Found: C, 55.29, H, 4.05; N, 14.55 .

5-/4-[3-chloro-3-trifluoromethyl-phenyl)-ureido]-phenoxy/pyrazine 2-carboxylic acid methylamide (6d) This compound was prepared by the reaction of $0.068 \mathrm{~g}(0.4 \mathrm{mmol})$ amide $4 \mathbf{a}$ and $0.132 \mathrm{~g}(0.4 \mathrm{mmol})$ urea 3c (Method A). After purification by column chromatography (mobile phase petrolether/ethyl acetate/methanol 2:2:0.1) and crystallization from ether, $0.093 \mathrm{~g}(50 \%)$ of a white solid was obtained; mp. $233-239^{\circ} \mathrm{C}$; IR (KBr): $\nu_{\max } 3415,3289$, $3134,3103,1710,1662,1608,1586,1550,1510,1487$, 1460, 1402, 1301, 1176, 1125, 1029, 837, 664, $509 \mathrm{~cm}^{-1}$; ${ }^{1} \mathrm{H}$ NMR (DMSO, $\left.600 \mathrm{MHz}\right): \delta=9.18(\mathrm{~s}, 1 \mathrm{H}, 14), 8.91(\mathrm{~s}$, $1 \mathrm{H}, 12), 8.69$ (s, 2H, 3, 1') 8.53 (s, 1H, 5), 8.12 (s, 1H, 20), $7.68-7.60$ (m, 2H, 16, 17), 7.54 (d, $J=8.73,2 \mathrm{H}, 8,10)$, $7.19(\mathrm{~d}, J=8.70,2 \mathrm{H}, 7,11), 2.82\left(\mathrm{~d}, J=4.56,3 \mathrm{H}, 2^{\prime}\right) ;{ }^{13} \mathrm{C}$ NMR (DMSO, $150 \mathrm{MHz}$ ): $\delta=162.94$ (4), 161.16 (1), 152.47 (13), 147.25 (6), 140.64 (5), 139.61 (15), 139.35 (2), 136.68 (9), 133.63 (3), 131.96 (17), 126.99-126.38 (q, 19, $J=30.48), 125.51-120.28(\mathrm{q}, 21, J=273.27), 123.10(16)$, 122.27 (18), 121.75 (8, 10), 120.04 (7, 11), 116.82-116.71 (q, 20, $J=5.44), 25.89\left(2^{\prime}\right)$; ESIMS $m / z$ (pos): 466.1, $\mathrm{C}_{20} \mathrm{H}_{16} \mathrm{ClF}_{3} \mathrm{~N}_{5} \mathrm{O}_{3}$ (calcd. 466.1); Anal. Calcd for $\mathrm{C}_{20} \mathrm{H}_{15} \mathrm{ClF}_{3} \mathrm{~N}_{5} \mathrm{O}_{3}$ : C, 51.57; H, 3.25; N, 15.03. Found: C, 51.22, H, 3.11; N, 15.44.

5-/4-[3-(4-chloro-3-trifluoromethyl-phenyl)-ureido]-phenoxy/-pyrazine-2-carboxylic acid (2-hydroxyethyl)amide (6e) This compound was prepared by the reaction of $0.087 \mathrm{~g}(0.4 \mathrm{mmol})$ amide $4 \mathbf{b}$ and $0.132 \mathrm{~g}(0.4 \mathrm{mmol})$ urea 3c (Method A). After purification by column chromatography (mobile phase ethyl acetate/methanol 2:0.1) and crystallization from methanol, $0.070 \mathrm{~g}(35 \%)$ of a white solid was obtained; mp. $177-180{ }^{\circ} \mathrm{C}$; IR (KBr): $\nu_{\max } 3437$, 3352, 3140, 2929, 1712, 1647, 1608, 1561, 1509, 1486, 1462, 1420, 1354, 1329, 1304, 1279, 1258, 1225, 1192, 1143, 1076, 1032, 1019, 915, 894, 845, 688, 664, 641, 513 $\mathrm{cm}^{-1} ;{ }^{1} \mathrm{H}$ NMR (DMSO, $\left.600 \mathrm{MHz}\right): \delta=9.26(\mathrm{~s}, 1 \mathrm{H}, 14)$, $9.00(\mathrm{~s}, 1 \mathrm{H}, 12), 8.70(\mathrm{~d}, J=1.30,1 \mathrm{H}, 3), 8.56(\mathrm{t}, J=5.85$, $\left.1 \mathrm{H}, 1^{\prime}\right), 8.54(\mathrm{~d}, J=1.31,1 \mathrm{H}, 5), 8.12(\mathrm{~d}, J=2.32,1 \mathrm{H}, 20)$, 7.68-7.59 (m, 2H, 16, 17), 7.56-7.51 (m, 2H, 8, 10), $7.22-7.17(\mathrm{~m}, 2 \mathrm{H}, 7,11), 4.77\left(\mathrm{t}, J=5.47,1 \mathrm{H}, 4^{\prime}\right)$, $3.56-3.50\left(\mathrm{q}, J=5.85,2 \mathrm{H}, 3^{\prime}\right), 3.41-3.35(\mathrm{q}, J=5.74,2 \mathrm{H}$, $\left.2^{\prime}\right) ;{ }^{13} \mathrm{C}$ NMR (DMSO, $\left.150 \mathrm{MHz}\right): \delta=162.39$ (4), 161.17 (1), 152.42 (13), 147.24 (6), 140.73 (5), 139.40 (15), 139.30 (2), 136.63 (9), 133.58 (3), 131.91 (17), 126.55 (t, 19, $J=$ 30.33), 125.46-120.03 (q, 21, $J=272.95), 123.01$ (16), 122.23 (18), 121.63 (8, 10), 120.01 (7, 11), 116.80-116.68 (q, 20, $J=5.68), 59.55\left(3^{\prime}\right), 41.48\left(2^{\prime}\right)$; ESIMS $m / z$ (pos): 518.2, $\mathrm{C}_{21} \mathrm{H}_{17} \mathrm{ClF}_{3} \mathrm{~N}_{5} \mathrm{NaO}_{4} \quad$ (calcd. 518.8), 496.8, $\mathrm{C}_{21} \mathrm{H}_{18} \mathrm{ClF}_{3} \mathrm{~N}_{5} \mathrm{O}_{4}$ (calcd. 496.8); Anal. Calcd for $\mathrm{C}_{21} \mathrm{H}_{17} \mathrm{ClF}_{3} \mathrm{~N}_{5} \mathrm{O}_{4}$ : C, 50.87; H, 3.46; N, 14.12. Found: C, 50.50, H, 3.41; N, 13.78 .

5-/4-[3-(4-chloro-3-trifluoromethylphenyl)-ureido]-phenoxy/-pyrazine-2-carboxylic acid benzyloxy-amide (6f)

This compound was prepared by the reaction of $0.112 \mathrm{~g}$ $(0.4 \mathrm{mmol})$ amide $4 \mathbf{f}$ and $0.132 \mathrm{~g}(0.4 \mathrm{mmol})$ urea $3 \mathbf{c}$ (Method A). After purification by column chromatography (mobile phase petrolether/ethyl acetate/methanol 2:2:0.1) and crystallization from ether/petrolether, $0.040 \mathrm{~g}(18 \%)$ of a white solid was obtained; mp. $208-212{ }^{\circ} \mathrm{C}$; IR (KBr): $\nu_{\max }$ $3351,3073,1678,1595,1547,1506,1486,1460,1420$, 1349, 1330, 1280, 1230, 1194, 1137, 1033, 1017, 912, 839, 753, 701, 664, 636, 532, $516 \mathrm{~cm}^{-1}$; ${ }^{1} \mathrm{H}$ NMR (DMSO, 600 $\mathrm{MHz}): \delta=12.10\left(\mathrm{~s}, 1 \mathrm{H}, 1^{\prime}\right), 9.20(\mathrm{~s}, 1 \mathrm{H}, 14), 8.94(\mathrm{~s}, 1 \mathrm{H}$, 12), 8.68 (d, $J=1.23,1 \mathrm{H}, 3), 8.52$ (d, $J=1.29,1 \mathrm{H}, 5), 8.12$ $(\mathrm{d}, J=2.28,1 \mathrm{H}, 20), 7.68-7.60(\mathrm{~m}, 2 \mathrm{H}, 16,17), 7.57-7.51$ (m, 2H, 8, 10), 7.49-7.45, 7.42-7.35 (2m, 5H, 4'-8'), $7.22-7.17(\mathrm{~m}, 2 \mathrm{H}, 7,11), 4.94\left(\mathrm{~s}, 2 \mathrm{H}, 2^{\prime}\right) ;{ }^{13} \mathrm{C} \mathrm{NMR}$ (DMSO, $150 \mathrm{MHz}$ ): $\delta=161.35$ (4), 160.17 (1), 152.48 (13), 147.15 (6), 140.93 (5), 139.35 (15), 138.83 (2), 136.74 (9), $135.73\left(3^{\prime}\right), 133.92$ (3), 131.99 (17), $128.78\left(4^{\prime}, 8^{\prime}\right)$, $128.28\left(5^{\prime}-7^{\prime}\right), \quad 128.24-117.38 \quad(q, \quad 18, \quad J=272.87)$, 127.30-126.09 (q, 19, $J=30.54), 123.07$ (16), 121.78 (8, 10), 120.05 (7, 11), 116.88-116.66 (q, 20, $J=5.53), 77.11$ (2'); ESIMS m/z (pos): 558.2, $\mathrm{C}_{26} \mathrm{H}_{20} \mathrm{ClF}_{3} \mathrm{~N}_{5} \mathrm{O}_{4}$ (calcd. 558.1); Anal. Calcd for $\mathrm{C}_{26} \mathrm{H}_{19} \mathrm{ClF}_{3} \mathrm{~N}_{5} \mathrm{O}_{4}$ : C, 55.97; $\mathrm{H}$, 3.43; N, 12.55. Found: C, 56.14, H, 3.40; N, 12.17.

5-/4-[3-(4-chloro-3-trifluoromethyl-phenyl)-ureido]-phenoxy/pyrazine-2-carboxylic acid (4-chloro-3-trifluoromethylphenyl)amide $(\mathbf{6 g})$ This compound was prepared by the reaction of $0.134 \mathrm{~g}(0.4 \mathrm{mmol})$ amide $4 \mathbf{e}$ and $0.132 \mathrm{~g}(0.4 \mathrm{mmol})$ urea $3 \mathbf{c}$ (Method A). After purification by column chromatography (mobile phase dichloromethane/methanol 9.5:0.5) and 
crystallization from ether/petrolether, $0.068 \mathrm{~g}(27 \%)$ of a white solid was obtained; $141-144^{\circ} \mathrm{C}$; IR (KBr): $\nu_{\max } 3353$, 3123, 1689, 1593, 1532, 1506, 1482, 1463, 1421, 1353, 1321, $1274,1261,1230,1185,1138,1113,1034,1020,835,665$ $\mathrm{cm}^{-1}$; ${ }^{1} \mathrm{H}$ NMR (DMSO, $\left.600 \mathrm{MHz}\right): \delta=11.09\left(\mathrm{~s}, 1 \mathrm{H}, 1^{\prime}\right)$, $9.20(\mathrm{~s}, 1 \mathrm{H}, 14) 8.95(\mathrm{~s}, 1 \mathrm{H}, 12), 8.86(\mathrm{~d}, J=1.26,1 \mathrm{H}, 3)$, $8.65(\mathrm{~d}, J=2.46,1 \mathrm{H}, 5), 8.24\left(\mathrm{dd}, J=2.40, J=6.42,1 \mathrm{H}, 3^{\prime}\right)$, $8.12\left(\mathrm{~d}, J=2.22,1 \mathrm{H}, 7^{\prime}\right), 7.73\left(\mathrm{~d}, J=8.89,1 \mathrm{H}, 6^{\prime}\right), 7.68-7.60$ (m, 2H, 16, 17), 7.58-7.53 (m, 2H, 8, 10), 7.26-7.21 (m, 2H, $7,11) ;{ }^{13} \mathrm{C}$ NMR (DMSO, $150 \mathrm{MHz}$ ): $\delta=161.97$ (4) 161.60 (1) 152.47 (13), 147.10 (6) 142.02 (5), 139.34 (15), 138.91 (2), 137.97 (2'), 136.83 (9), 133.76 (3), 131.98 (17), 131.96 (6'), 128.23-117.37 (q, 21, $J=273.33), 128.18-117.32$ (q, $J$ $\left.=273.33,8^{\prime}\right), 127.30-126.08(\mathrm{q}, J=30.59,19), 127.24$ $-126.02\left(\mathrm{q}, J=30.77,4^{\prime}\right), 125.26(16) 124.71\left(\mathrm{~d}, J=1.66,5^{\prime}\right)$ $123.06\left(7^{\prime}\right), 122.29(\mathrm{~d}, J=1.65,18), 121.79(8,10), 120.04$ (7, 11) $119.44-119.21$ (q, $J=5.71,20), 116.88-116.65$ (q, 3', $J$ = 5.71); ESIMS m/z (pos): 630.2, $\mathrm{C}_{26} \mathrm{H}_{15} \mathrm{Cl}_{2} \mathrm{~F}_{6} \mathrm{~N}_{5} \mathrm{O}_{3}$ (calcd. 630.3); Anal. Calcd for $\mathrm{C}_{26} \mathrm{H}_{15} \mathrm{Cl}_{2} \mathrm{~F}_{6} \mathrm{~N}_{5} \mathrm{O}_{3}$ : C, 49.54; H, 2.40; N, 11.11. Found: C, 49.75, H, 2.76; N, 10.83 .

5-/4-[3-(4-chloro-3-trifluoromethyl-phenyl)-ureido]-phenoxy/-pyrazine-2-carboxylic acid cyclopentylamide (6h) This compound was prepared by the reaction of $0.090 \mathrm{~g}$ $(0.4 \mathrm{mmol})$ amide $4 \mathrm{c}$ and $0.132 \mathrm{~g}(0.4 \mathrm{mmol})$ urea $\mathbf{3 c}$ (Method A). After crystallization from ether, $0.094 \mathrm{~g} \mathrm{(45 \% )}$ of a white solid was obtained. Product $\mathbf{6 h}$ was also prepared by the reaction of $0.060 \mathrm{~g}(0.2 \mathrm{mmol})$ compound 5a and $0.066 \mathrm{~g}$ (0.3 mmol) 4-chloro-3-(trifluoromethyl)phenylisocyanate (Method B). After crystallization from ether $0.051 \mathrm{~g}$ (49\%) of pure product was obtained; mp. 231-233 ${ }^{\circ} \mathrm{C}$; IR $(\mathrm{KBr}): \nu_{\max } 3382,3294,3115,3081,2963,2875,1711$, 1658, 1604, 1549, 1509, 1485, 1460, 1327, 1195, 1133, 1023, 840, 661, $513 \mathrm{~cm}^{-1} ;{ }^{1} \mathrm{H}$ NMR (DMSO, $600 \mathrm{MHz}$ ): $\delta$ $=9.20(\mathrm{~s}, 1 \mathrm{H}, 14), 8.95(\mathrm{~s}, 1 \mathrm{H}, 12), 8.69(\mathrm{~d}, J=1.20,1 \mathrm{H}$, 3), $8.53(\mathrm{~d}, J=1.26,1 \mathrm{H}, 5), 8.49\left(\mathrm{~d}, J=7.98,1 \mathrm{H}, 1^{\prime}\right), 8.12$ $(\mathrm{d}, J=2.46,1 \mathrm{H}, 20), 7.67-7.65(\mathrm{~m}, 1 \mathrm{H}, 16), 7.63-7.61(\mathrm{~m}$, 1H, 17), 7.55-7.53 (m, 2H, 8, 10), 7.21-7.18 (m, 2H, 7, 11), 4.27-4.22 (m, 1H, 2'), 1.91-1.85 (m, 2H, 3'), $1.72-1.68\left(\mathrm{~m}, 2 \mathrm{H}, 6^{\prime}\right), 1.62-1.53\left(\mathrm{~m}, 4 \mathrm{H}, 4^{\prime}, 5^{\prime}\right) ;{ }^{13} \mathrm{C} \mathrm{NMR}$ (DMSO, $150 \mathrm{MHz}$ ): $\delta=162.02$ (4), 161.14 (1), 152.48 (13), 147.28 (6), 140.84 (5), 139.70 (15), 139.35 (2), 136.67 (9), 133.51 (3), 131.97 (17), 126.99-126.39 (q, $J=30.50$, 19), 125.52-120.09 (q, $J=273.44,21), 123.05$ (16), 122.28 (18), $121.71(8,10), 120.04(7,11), 116.81-116.70$ (q, $J=$ 4.39, 20), $50.50\left(2^{\prime}\right), 32.01\left(3^{\prime}, 6^{\prime}\right), 23.52$ (4', 5'); ESIMS $\mathrm{m} / z$ (pos): 520.1, $\mathrm{C}_{24} \mathrm{H}_{22} \mathrm{ClF}_{3} \mathrm{~N}_{5} \mathrm{O}_{3}$ (calcd. 520.1); Anal. Calcd for $\mathrm{C}_{24} \mathrm{H}_{21} \mathrm{ClF}_{3} \mathrm{~N}_{5} \mathrm{O}_{3}$ : C, 55.44; $\mathrm{H}, 4.07$; N, 13.47. Found: C, 55.10, H, 3.77; N, 13.10.

5-/4-[3-(4-chloro-3-trifluoromethyl-phenyl)-ureido]-phenoxy/-pyrazine-2-carboxylic acid cyclohexylamide (6i) This compound was prepared by the reaction of $0.096 \mathrm{~g}$ $(0.4 \mathrm{mmol})$ amide $4 \mathbf{d}$ and $0.132 \mathrm{~g}(0.4 \mathrm{mmol})$ urea $3 \mathbf{c}$
(Method A). After several crystallizations from ether, 0.135 g $(63 \%)$ of a white solid was obtained. Product $6 \mathbf{i}$ was also prepared by the reaction of $0.062 \mathrm{~g}(0.2 \mathrm{mmol})$ compound 5b and $0.066 \mathrm{~g}(0.3 \mathrm{mmol})$ 4-chloro-3-(trifluoromethyl) phenylisocyanate (Method B). After crystallization from ether, $0.065 \mathrm{~g}(61 \%)$ of pure product was obtained; mp. 217-220 ${ }^{\circ} \mathrm{C}$; IR (KBr): $\nu_{\max } 3394,3322,3122,3082,2928$, 2857, 1711, 1660, 1595, 1513, 1481, 1421, 1310, 1227, 1143, 1033, 844, 576, $515 \mathrm{~cm}^{-1}$; ${ }^{1} \mathrm{H}$ NMR (DMSO, 600 $\mathrm{MHz}): \delta=9.19(\mathrm{~s}, 1 \mathrm{H}, 14), 8.93(\mathrm{~s}, 1 \mathrm{H}, 12), 8.69(\mathrm{~d}, J=$ $1.20,1 \mathrm{H}, 3), 8.52(\mathrm{~d}, J=1.20,1 \mathrm{H}, 5), 8.40(\mathrm{~d}, J=8.52,1 \mathrm{H}$, $\left.1^{\prime}\right), 8.12(\mathrm{~d}, J=2.13,1 \mathrm{H}, 20), 7.68-7.60(\mathrm{~m}, 1 \mathrm{H}, 16,17)$, 7.56-7.51 (m, 2H, 8, 10), 7.22-7.17 (m, 2H, 7, 11), 3.84-3.73 (m, 1H, 2' $), 1.80-1.70,1.62-1.58,1.48-1.25$, 1.19-1.07 (4m, 10H, $\left.3^{\prime}-7^{\prime}\right) ;{ }^{13} \mathrm{C}$ NMR (DMSO, $150 \mathrm{MHz}$ ): $\delta=161.43$ (4), 161.17 (1), 152.48 (13), 147.30 (6) 140.90 (5) 139.67 (15) 139.36 (2), 136.68 (9) 133.55 (3), 131.99 (17) $128.25-117.41$ (q, $J=273.26,21) 127.31-126.09$ (q, $J=30.29,19), 123.54$ (16), 122.27 (18), 122.18 (8, 10), $120.53(7,11)$ 116.88-116.65 (q, $J=5.82,20), 47.93\left(2^{\prime}\right)$, $32.13\left(3^{\prime}, 7^{\prime}\right), 25.08\left(5^{\prime}\right), 24.82\left(4^{\prime}, 6^{\prime}\right)$; ESIMS $m / z$ (pos): 534.4, $\mathrm{C}_{25} \mathrm{H}_{24} \mathrm{ClF}_{3} \mathrm{~N}_{5} \mathrm{O}_{3}$ (calcd. 534.9); Anal. Calcd for $\mathrm{C}_{25} \mathrm{H}_{23} \mathrm{ClF}_{3} \mathrm{~N}_{5} \mathrm{O}_{3}$ : C, 56.24; $\mathrm{H}, 4.34 ; \mathrm{N}, 13.12$. Found: $\mathrm{C}$, 56.65, H, 4.01; N, 12.74 .

\section{Biological evaluation}

\section{Antiproliferative evaluation assay}

The cell lines (CEM, HeLa, A549, HepG2, SW620, MCF7, Molt4/C8, L1210,WI-38) were cultured as monolayers and maintained in Dulbecco's modified Eagle medium (DMEM) supplemented with $10 \%$ fetal bovine serum (FBS), $2 \mathrm{mM}$ L-glutamine, $100 \mathrm{U} / \mathrm{mL}$ penicillin, and 100 $\mu \mathrm{g} / \mathrm{mL}$ streptomycin in a humidified atmosphere with $5 \%$ $\mathrm{CO}_{2}$ at $37^{\circ} \mathrm{C}$. The cell lines were inoculated onto a series of standard 96-well microtiter plates on day 0 at seeding density of 3000-6000 cells per well depending upon their specific doubling times. Freshly prepared dilutions of test compounds in culture medium in the concentration range from $10^{-8}$ to $10^{-4} \mathrm{M}$ were added to the microtiter plates, and the cells were grown for further 3-4 days. Working dilutions of the compounds were freshly prepared on the day of testing. The solvent (DMSO) was also tested for its potential inhibitory activity by adjusting its concentration to the values used for the preparation of the working concentrations (DMSO concentration never exceeded $0.1 \%$ ). After 3-4 days of incubation, the cell growth rate was evaluated first light microscopically, then by performing the MTT [3(4,5-dimethylthiazol-2-yl)-2,5-diphenyltetrazolium bromide] assay. Experimentally determined absorbance values were transformed into the cell percentage of growth (PG) using the formulas proposed by $\mathrm{NIH}$ and described 
previously (Gazivoda et al., 2008). This method directly relies on control cells behaving normally at the day of assay commencement because it compares the growth of treated cells with that of untreated cells in control wells on the same plate. The results are therefore a percentile difference from the calculated expected value. The $\mathrm{IC}_{50}$ values for each compound are calculated from dose-response curves using linear regression analysis by fitting the mean test concentrations that give PG values above and below the reference value. However, if all of the tested concentrations produce PGs exceeding the respective reference level of effect (e.g., PG value of 50) for a given cell line, then the highest tested concentration is assigned as the default value (in the screening data report that default value is preceded by a ">" sign). Each test point was performed in quadruplicate in three individual experiments. The results were statistically analyzed (Analysis of variance, Tukey post hoc test at $p<0.05$ ).

\section{Determination of c-Raf activity using Western blotting}

In order to study the effects of selected compounds on c-Raf activity, known protein target of multikinase inhibitor sorafenib, cells were cultured in 6-well plates at seeding density of $2 \times 10^{5}$ cells/well and subjected to treatment for indicated time points. Cells were lysed in RIPA buffer containing $20 \mathrm{mM}$ Tris- $\mathrm{HCl}$ ( $\mathrm{pH} 7.5$ ), $150 \mathrm{mM} \mathrm{NaCl}, 1 \mathrm{mM}$ $\mathrm{Na}_{2}$ EDTA, $1 \mathrm{mM}$ ethyleneglycol tetraacetic acid (EGTA), $1 \%$ NP-40, and $1 \%$ sodium deoxycholate supplemented with protease inhibitor cocktail (Roche). Total proteins (50 $\mu \mathrm{g}$ ) were resolved on $12 \%$ Tris-glycine polyacrylamide gels and transferred to polyvinylidene fluoride (PVDF) membranes. Subsequently, membranes were blocked for $1 \mathrm{~h}$ at room temperature with $4 \%$ bovine serum albumin (BSA) in TBST $[50 \mathrm{mmol} / \mathrm{L}$ Tris base, $150 \mathrm{mM} \mathrm{NaCl}, 0.1 \%$ Tween $20(\mathrm{pH} 7.5)]$ and probed overnight at $4{ }^{\circ} \mathrm{C}$ with primary antibody against Phospho-c-Raf (Ser259) (Cell Signaling Technology; dilution 1:500). Membranes were washed with TBST and incubated with an anti-rabbit (DakoCytomation) horseradish peroxidase-conjugated secondary antibody at room temperature for $1 \mathrm{~h}$. Individual proteins were visualized by the BM Chemiluminescence Western Blotting Substrate (POD) (Roche, Switzerland).

\section{Conclusions}

Nine novel pyrazine sorafenib analogs $\mathbf{6 a}-\mathbf{i}$ differing in amide and/or urea regions were prepared and evaluated as potential cytostatic agents. The strongest cytostatic activity to all tested cell lines was observed for compound $\mathbf{6 g}$, followed by $\mathbf{6 e}$, $\mathbf{6 f}$, and $\mathbf{6 i}$, all having 4-chloro-3trifluoromethylphenyl moiety in urea part. Their antiproliferative activity, as well as cytotoxicity to normal human fibroblasts cells, was comparable to sorafenib. Compounds $\mathbf{6 c}$ and $\mathbf{6 h}$ exerted cytostatic activities that surpassed the effects observed with sorafenib in three cancer cell lines (HepG2, HeLa, A549). Similar to sorafenib, compound $\mathbf{6 h}$ proved to be cytotoxic to normal human fibroblast cells, whereas compound $\mathbf{6 c}$ did not diminish proliferative capacity of these cells. Antiproliferative activity of $\mathbf{6 h}$ could be, at least partially, attributed to its inhibitory effect on c-Raf activation. On contrary, $\mathbf{6 c}$ did not inhibit the activity of c-Raf, which implies that other cell signaling pathways govern its antiproliferative effects. In conclusion, due to its reduced adverse effects on the growth of normal cells and more potent anticancer activity in particular cancer cell lines in comparison with sorafenib, compound 6c, e.g., 5-/4-[3-(4-bromophenyl)-ureido]-phenyloxy/-pyrazine-2-carboxylic acid cyclopentylamide, may be considered a potential scaffold for development of new, more effective, and safer drugs for treating hepatocellular, lung, and cervical carcinoma.

Acknowledgments Support for this study by Croatian Science Foundation (project number IP-2014-09-1501), University of Zagreb and University of Rijeka (Projects numbers BM1.9, 13.11.2.1.12 and 13.11.1.1.11), Republic of Croatia and the KU Leuven, Belgium (GOA 10/014), are gratefully acknowledged. We sincerely thank Lizette Van Berckelaer for performing the cytostatic in vitro experiments.

\section{Compliance with ethical standards}

Conflict of interest The authors declare that they have no conflict of interest.

Open Access This article is distributed under the terms of the Creative Commons Attribution 4.0 International License (http://creativecommons.org/licenses/by/4.0/), which permits unrestricted use, distribution, and reproduction in any medium, provided you give appropriate credit to the original author(s) and the source, provide a link to the Creative Commons license, and indicate if changes were made.

\section{References}

Babić Ž, Crkvenčić M, Rajić Z, Mikecin A-M, Kralj M, Balzarini J, Petrova M, Vanderleyden J, Zorc B (2012) New sorafenib derivatives: synthesis, antiproliferative activity against tumour cell lines and antimetabolic evaluation. Molecules 17:1124-1137

Butula I, Proštenik MV, Vela V (1977) Reactions with 1-benzotriazole carboxylic acid chloride. I.: synthesis of the 2,6-bis (hydroxymethyl)pyridine dicarbamates. Croat Chem Acta 49:837-842

Butula I, Vela V, Ivezić B (1978) Reaktionen mit 1-benzotriazol carbonsaurechlorid.IV.Synthese von substituierten Harnstoffen, Semicarbaziden und Carbaziden. Croat Chem Acta 51:339-346

Butula I, Jadrijević-Mladar Takač M (2000) Reaction with 1benzotriazolecarboxylic acid chloride. VIII.: synthesis of $\mathrm{N}$ hydroxyisocyanate derivatives. Croat Chem Acta 73:569-574 
Chong CR, Jänne PA (2013) The quest to overcome resistance to EGFR-targeted therapies in cancer. Nat Med 19:1389-400

Crona DJ, Keisler MD, Walko CM (2013) Regorafenib: a novel multitargeted tyrosine kinase inhibitor for colorectal cancer and gastrointestinal stromal tumors. Ann Pharmacother 47:1685-1696

Gazivoda T, Raić-Malić S, Krištafor V, Makuc D, Plavec J, Bratulic S, Kraljević Pavelić S, Pavelić K, Naesens L, Andrei G, Snoeck R, Balzarini J, Mintas M (2008) Synthesis, cytostatic and anti-HIV evaluations of the new unsaturated acyclic C-5 pyrimidine nucleoside analogues. Bioorg Med Chem 16:5624-5634

Holohan C, Van Schaeybroeck S, Longley DB, Johnston PG (2013) Cancer drug resistance: an evolving paradigm. Nat Rev Cancer 13:714-726

Jiao Y, Xin BT, Zhang Y, Wu J, Lu X, Zheng Y, Tang W, Zhou X (2015) Design, synthesis and evaluation of novel 2-( $1 H$-imidazol2-yl) pyridine sorafenib derivatives as potential BRAF inhibitors and anti-tumor agents. Eur J Med Chem 90:170-183

Lu C, Tang K, Li Y, Li P, Lin Z, Yin D, Chen X (2014) Design, synthesis and evaluation of novel diaryl urea derivatives as potential antitumor agents. Eur J Med Chem 77:351-360

Luzina EL, Popov AV (2012) Synthesis, evaluation of anticancer activity and COMPARE analysis of $N$-bis(tri fluoromethyl)alkyl$N$ '-substituted ureas with pharmacophoricmoieties. Eur J Med Chem 53:364-373

Qin M, Zhai X, Xie H, Ma J, Lu K, Wang Y, Wang L, Gu Y, Gong P (2014) Design and synthesis of novel 2-(4-(2-(dimethylamino) ethyl)-4H-1,2,4-triazol-3-yl)pyridines as potential antitumor agents. Eur J Med Chem 81:47-58

Schutz FA, Je Y, Choueri TK (2011) Hematologic toxicities in cancer patients treated with the multi-tyrosine kinase sorafenib: a meta-analysis of clinical trials. Crit Rev Oncol Hematol 80:291-300

Sun M, Wu X, Chen J, Cai J, Cao M, Ji M (2010) Design, synthesis, and in vitro antitumor evaluation of novel diaryl ureas derivatives. Eur J Med Chem 45:2299-2306
Villaneuva A, Llovet JM (2012) Second-line therapies in hepatocellular carcinoma: emergence of resistance to sorafenib. Clin Cancer Res 18:1824-1826

Wan PTC, Garnett MJ, Roe SM, Lee S, Niculescu-Duvaz D, Good VM (2004) Mechanism of activation of the RAF-ERK signaling pathway by oncogenic mutations of B-Raf. Cell 116:855-867

World Health Organization (2015) Cancer: key facts. Available at: http://www.who.int/mediacentre/factsheets/fs297/en/

Wilhelm S, Carter C, Lynch M, Lowinger T, Dumas J, Smith RA, Schwartz B, Simantov R, Kelley S (2006) Discovery and development of sorafenib: a multikinase inhibitor for treating cancer. Nat Rev Drug Discov 5:835-844

Wilhelm SM, Adnane L, Newell P, Villanueva A, Llovet JM, Lynch M (2008) Preclinical overview of sorafenib, a multikinase inhibitor that targets both Raf and VEGF and PDGF receptor tyrosine kinase signaling. Mol Cancer Ther 7:3129-3140

Winum J-Y, Carta F, Ward C, Mullen P, Harrison D, Langdon SP, Cecchi A, Scozzafava A, Kunkler I, Supuran CT (2012) Ureidosubstituted sulfamates show potent carbonic anhydrase IX inhibitory and antiproliferative activities against breast cancer cell lines. Bioorg Med Chem Lett 22:4681-4685

Yang LL, Li GB, Ma S, Zou C, Zhou S, Sun QZ, Cheng C, Chen X, Wang LJ, Feng S, Li LL, Yang SY (2013) Structure-activity relationship studies of pyrazolo[3,4-d]pyrimidine derivatives leading to the discovery of a novel multikinase inhibitor that potently inhibits FLT3 and VEGFR2 and evaluation of its activity against acute myeloid leukemia in vitro and in vivo. J Med Chem 56:1641-1655

Yao J, He Z, Chen J, Sun W, Fang H, Xu W (2012) Design, synthesis and biological activities of sorafenib derivatives as antitumor agents. Bioorg Med Chem Lett 22:6549-6553

Zhan W, Li Y, Huang W, Zhao Y, Yao Z, Yu S, Yuan S, Jiang F, Yao S, Li S (2012) Design, synthesis and antitumor activities of novel bis-aryl ureas derivatives as Raf kinase inhibitors. Bioorg Med Chem 20:4323-4239 